\title{
DESIGN, FABRICATION, AND CHARACTERIZATION OF A MAGNETIC POROUS PDMS AS AN ON-DEMAND DRUG DELIVERY DEVICE
}

by

\author{
Ali Shademani \\ B.Sc., University of Tehran, 2013 \\ A THESIS SUBMITTED IN PARTIAL FULFILLMENT OF \\ THE REQUIREMENTS FOR THE DEGREE OF \\ MASTER OF APPLIED SCIENCE \\ in \\ THE FACULTY OF GRADUATE AND POSTDOCTORAL STUDIES \\ (Mechanical Engineering) \\ THE UNIVERSITY OF BRITISH COLUMBIA \\ (Vancouver)
}

August 2016

(C) Ali Shademani, 2016 


\begin{abstract}
We introduce a novel, on-demand drug delivery device based on a biocompatible magnetic sponge. The sponge is made of a porous polydimethylsiloxane (PDMS) mixed with carbonyl iron (CI) particles. The sponge is deformed under a magnetic field and consequently leads to releasing its contents. As a proof of concept study, three different CI/PDMS wt\% ratios of 50\%, $100 \%$, and $150 \%$ were selected where, the $100 \%$ showed the most deformation under various magnetic fields. Although this sponge can solely be used as a potential drug delivery agent, a separate reservoir has been fabricated to protect the sponge and control the release rate. The final device has a diameter of $6 \mathrm{~mm}$ with a thickness of $2 \mathrm{~mm}$. Controlled release of methylene blue (MB) and docetaxel (DTX) have been investigated to demonstrate the consistency and flexibility in adjusting the release rate from the device to suit different treatment requirements. Ex vivo tissue implantation has also been accomplished. This device is able to be implanted and deliver therapeutic agents at prescribed dosages.
\end{abstract}




\section{Preface}

The research presented in this dissertation was carried out at the University of British Columbia under the supervision of Dr. Mu Chiao in the Department of Mechanical Engineering. A version of this work was also presented in the following conference:

- A. Shademani, H. Zhang, J. K. Jackson, H. M. Burt and M. Chiao, “A Magnetic Sponge as an On-demand Drug Delivery Device”, Scientific and Clinical Applications of Magnetic Carriers, British Columbia, Vancouver, Canada, 2016

All facets of this project including literature review, magnetic sponge fabrication, drug delivery device characterization, controlled drug release studies, and tissue implantation have been performed by the author of this thesis. The author wrote this thesis, and carried out experiments under the supervision of Dr. Chiao. Guidance, expert advice, and suggested edits of Dr. Chiao helped the author to accomplish the tasks. Dr. Burt provided the author with the necessary equipment for drug release tests. Mr. Jackson from Dr. Burt's group at pharmaceutical science department assisted the author with equipment and technical advice during evaluation of drug delivery device. Dr. Zhang from Microelectromechanical system laboratory (MEMS lab.) provided the author with expert advice and technical guidance during the whole course of this project. Mr. Zachkani from MEMS lab provided the author with crucial training and advice.

The author's major contributions in this thesis are listed below:

1. Characterization and modifying the fabrication process of a biocompatible magnetic sponge which can be used as drug delivery agent.

2. Developing a novel magnetic drug delivery device based on a magnetic porous polymer which is powerless and is able to release the drug on-demand with adjustable dosage. 
3. Developing the conceptual design and fabrication process of this new drug delivery device.

4. Device characterization and numerical simulation of released drug behaviour

5. Acquiring reproducible and adjustable release profiles with consistent trends

6. Demonstrating the biological performance of the proposed device ex vivo, and providing drug release behaviour while the device is implanted in a tissue. 


\section{Table of Contents}

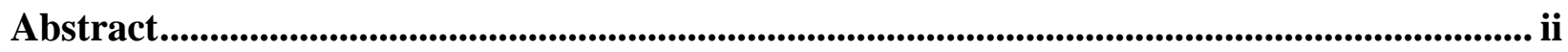

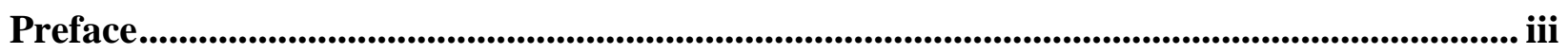

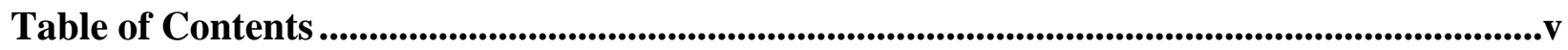

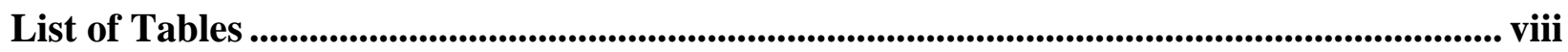

List of Figures............................................................................................................................................... ix

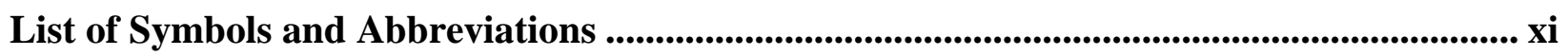

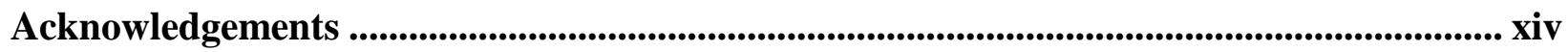

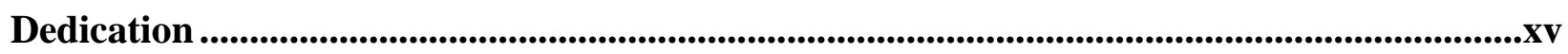

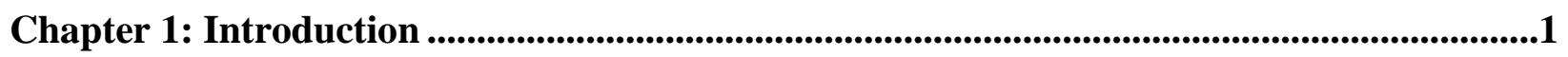

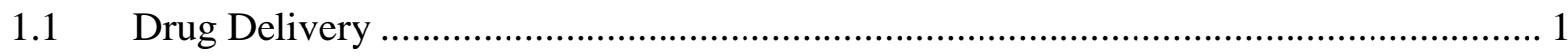

1.2 Controlled Drug Release System ................................................................ 2

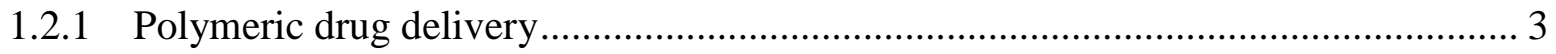

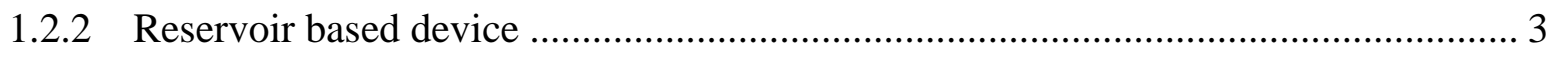

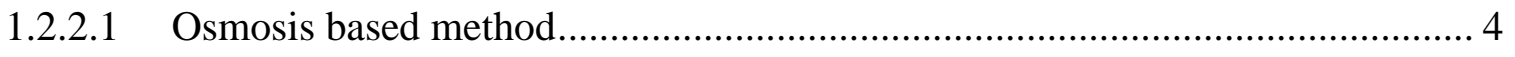

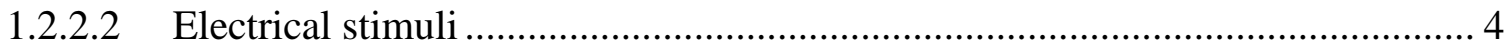

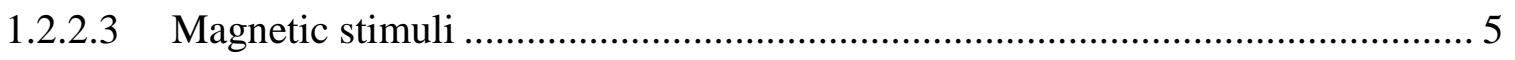

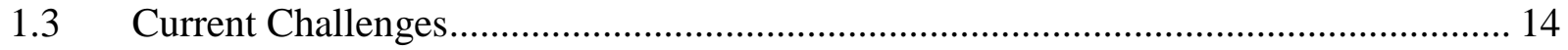

1.4 Magnetic Sponge as an On-Demand Drug Delivery Device ................................... 15

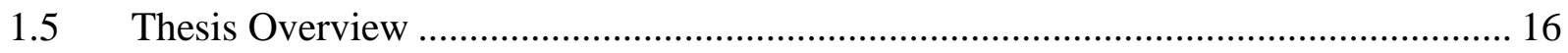

Chapter 2: Development and Characterisation of the Magnetic Porous PDMS Drug

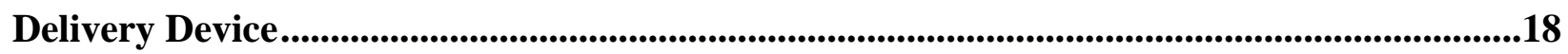




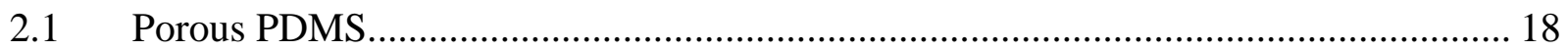

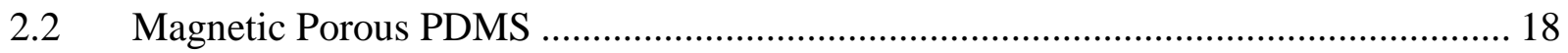

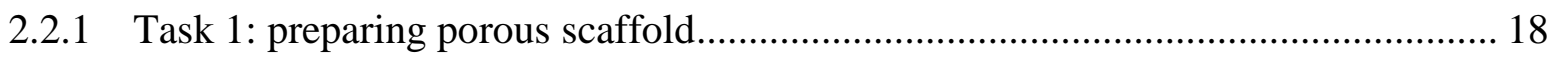

2.2.2 Task 2: Magnetic PDMS ..................................................................................... 19

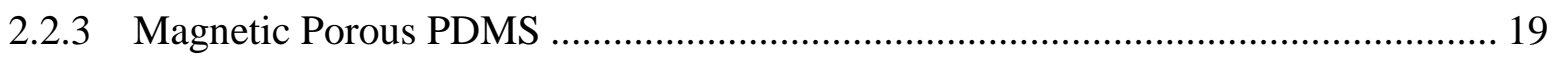

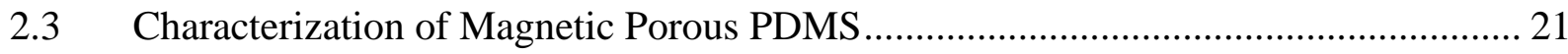

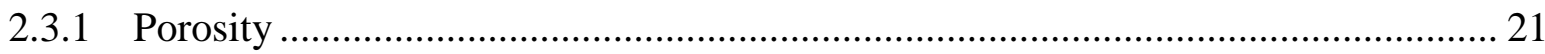

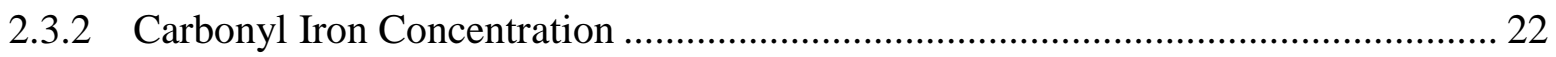

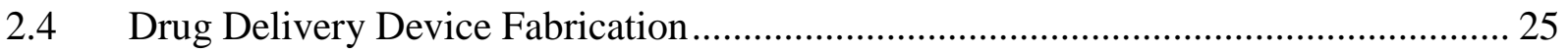

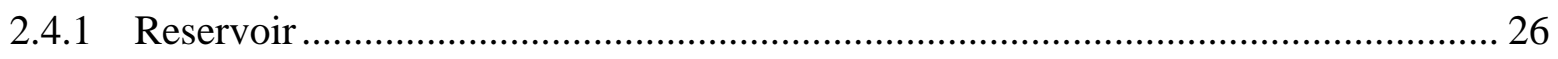

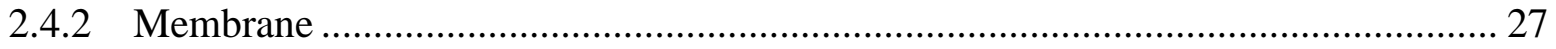

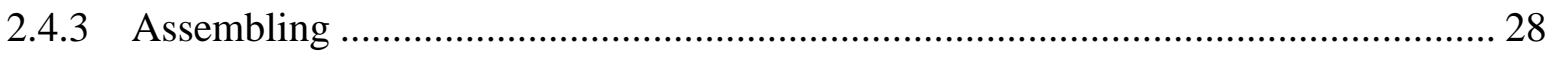

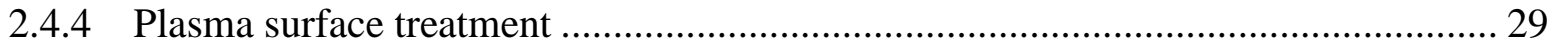

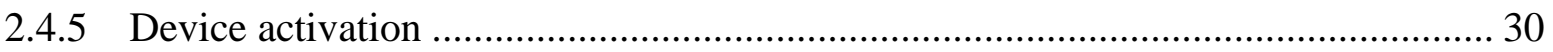

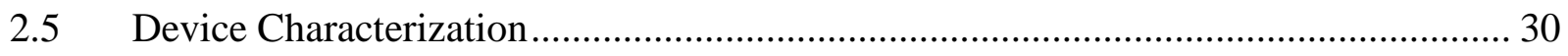

2.5.1 Methylene blue release ………………............................................................. 30

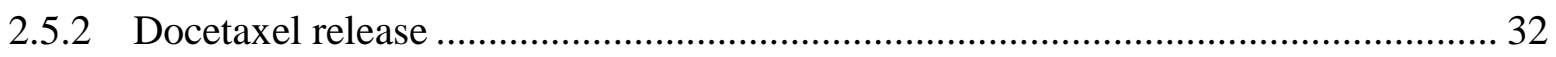

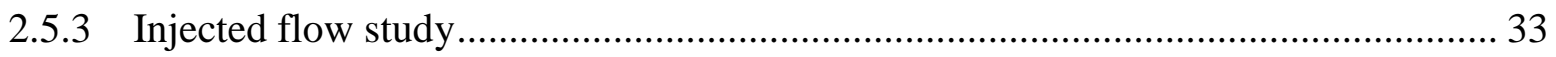

2.5.4 Ex vivo tissue implantation................................................................................... 34

Chapter 3: Results and Discussions............................................................................................35

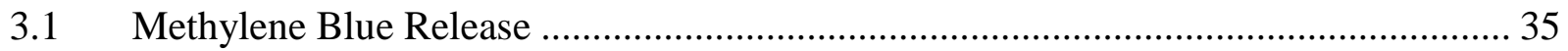

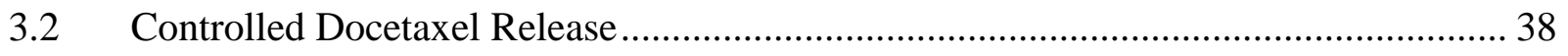

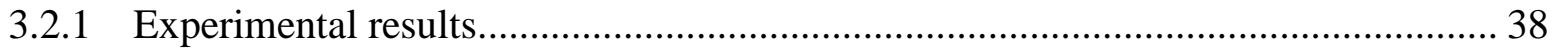




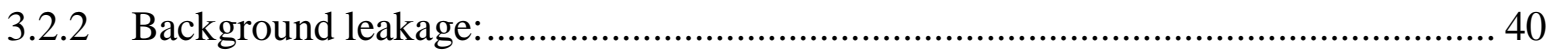

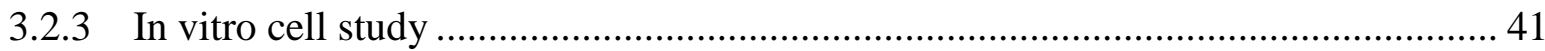

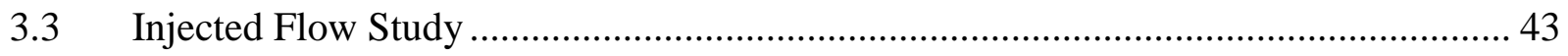

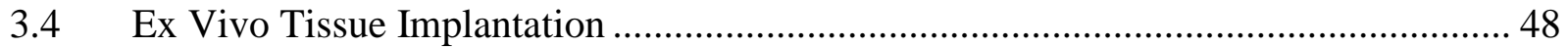

Chapter 4: Conclusions and Future Work …..............................................................................50

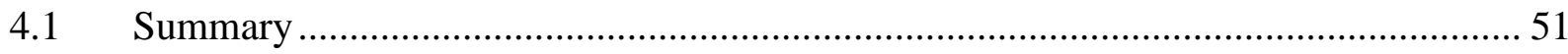

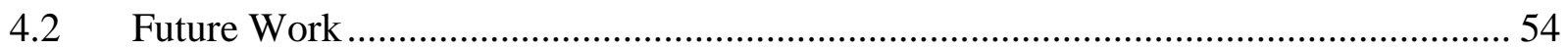

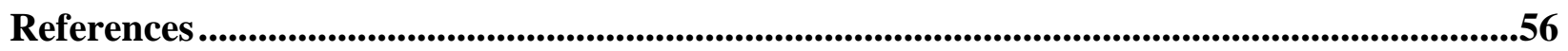

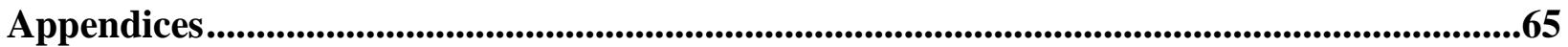

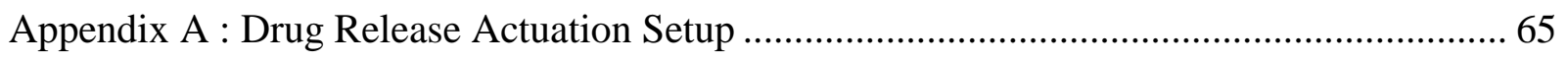

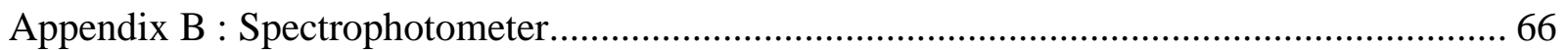




\section{List of Tables}

Table 1- Summarized reservoir based drug delivery methods............................................... 7

Table 2- Estimated shear stress for different magnetic field strengths of $97.4 \mathrm{mT}, 117.2 \mathrm{mT}$, and

$114.7 \mathrm{mT}$ 47 


\section{List of Figures}

Figure 1- Function of different types of drug delivery according to therapeutic window [10] ...... 2

Figure 2- schematic view of the device components ...................................................... 15

Figure 3- From right to left; magnetic porous PDMS scaffold, sample punched from the scaffold, and the consequent magnetic sponge after submerging the sample in water 20

Figure 4- a) Sponge, b) Cross section of the magnetic porous PDMS, c) Different CI to PDMS

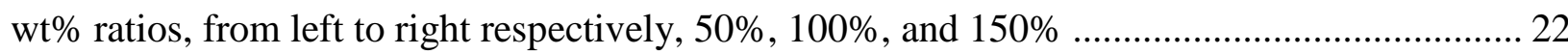

Figure 5- Displacement of sponges with different CI concentration in various magnetic fields . 23 Figure 6- Stress versus strain for different sponges with $0,50 \%, 100 \%$, and $150 \%$ CI/PDMS wt $\%$ ratios. 24

Figure 7- Mold on the left side and the demolded PDMS layer (right side) out of which one sample reservoir was punched

Figure 8- Real device, in comparison with one Canadian dollar. This device is also roughly as

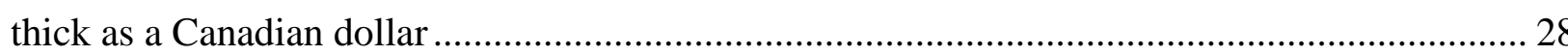

Figure 9- SEM image from cross section of the proposed drug delivery device....................... 29

Figure 10- Methylene blue release, a) dormant phase, b) actuation ..................................... 31

Figure 11- Cumulative methylene blue release over time .............................................. 36

Figure 12- MB release in various magnetic field strength................................................ 37

Figure 13- Cumulative docetaxel release in a $180 \mathrm{mT}$ magnetic field strentgh ....................... 39

Figure 14- Three consecutive day docetaxel release ....................................................... 40

Figure 15- Inhibition of PC3 cell proliferation using docetaxel [30] .................................... 42

Figure 16- Position of tip of injected flow versus time ................................................. 44 
Figure 17- Estimated tip velocity of the jet flow versus time.

Figure 18- Schematic view of the injected MB and involved parameters.

Figure 19- a) Implanted device inside a porcine bladder tissue, before and after actuation are shown, the blue stain is caused by the methylene blue loaded in the reservoir in addition to docetaxel, b) Measured docetaxel in the targeted site of the tissues after 2 and 4 times actuation

Figure 20- Actuation setup for drug release test ......................................................... 65

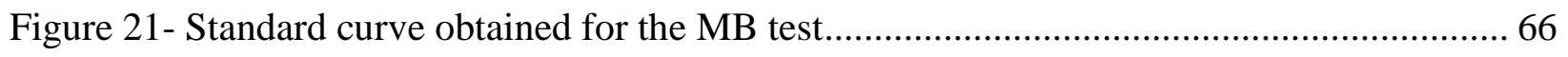

Figure 22- UV-Vis spectrophotometer (50 BIO, Varian Medical Systems Inc., Palo Alto, CA,

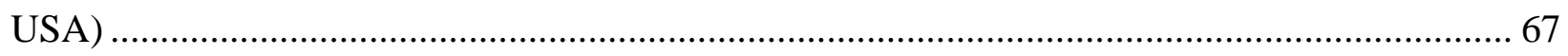




\section{List of Symbols and Abbreviations}

\begin{tabular}{|c|c|}
\hline$\% \mathrm{w} / \mathrm{v}$ & weight (grams) to volume (millilitre) percentage \\
\hline$\% \mathrm{w} / \mathrm{w}$ & weight to weight percentage \\
\hline $\mathrm{wt} \%$ & weight to weight percentage \\
\hline${ }^{\circ} \mathrm{C}$ & degree Celsius \\
\hline B & magnetic flux density \\
\hline BSA & bovine serum albumin \\
\hline $\mathrm{Ci}$ & curie \\
\hline $\mathrm{cm}$ & centimeter \\
\hline CI & carbonyl iron \\
\hline dia. & diameter \\
\hline DCM & dichloromethane \\
\hline DPM & disintegration per minute \\
\hline DTX & docetaxel \\
\hline $\mathrm{E}$ & elasticity modulus \\
\hline IPA & isopropanol \\
\hline IR & Infrared \\
\hline ID & Inner diameter \\
\hline MB & methylene blue \\
\hline MEMS & microelectromechanical systems \\
\hline $\mathrm{mg}$ & milligrams \\
\hline $\mathrm{mm}$ & millimeter \\
\hline
\end{tabular}




\begin{tabular}{|c|c|}
\hline $\mathrm{ml}$ & milliliter \\
\hline $\mathrm{mT}$ & millitesla \\
\hline $\min$ & minute \\
\hline $\mathrm{kPa}$ & kilopascal \\
\hline NIR & near infrared \\
\hline $\mathrm{NCI}$ & national cancer institute \\
\hline ng & nanogram \\
\hline $\mathrm{nl}$ & nanoliter \\
\hline OD & Outer diameter \\
\hline $\mathrm{Pa}$ & Pascal \\
\hline PAA & poly(acrylic acid) \\
\hline PBS & phosphate buffered saline \\
\hline PDMS & poly(dimethylsiloxane) \\
\hline $\mathrm{pH}$ & measure of acidity or basicity \\
\hline $\mathrm{rpm}$ & revolutions per minute \\
\hline $\mathrm{s}$ & second \\
\hline SEM & scanning electron microscope \\
\hline $\mathrm{T}$ & tesla \\
\hline $\mathrm{t}$ & time \\
\hline UV & ultraviolet \\
\hline V & flow velocity \\
\hline$\mu l$ & microliter \\
\hline
\end{tabular}


$\mu \mathrm{m}$

$\mu$

W

$\tau$ micrometer

dynamic viscosity

maximum width of injected methylene blue

shear stress 


\section{Acknowledgements}

First of all, I would like to acknowledge the support and priceless advice of my supervisor, Dr. Mu Chiao, who gave me this valuable opportunity to work in his lab and on this fascinating project. He always encourages me to put my ideas into practice and guides me along this path. I would like to express my gratitude to Mr. John Jackson from the pharmaceutical science department who always motivated me and his precious comments and suggestions were paramount in the accomplishment of this study. I would like to thank my colleague and friend Payam Zachkani for training and helping me with his invaluable experience. I appreciate the time and effort of my colleague Dr. Hongbin Zhang and his technical advice which led to progress of this project.

I would also like to thank Dr. Hong Ma, Dr. Karen Cheung, and Dr. Helen Burt for allowing me to use their lab space and equipment. I am also grateful to have Dr. Hong Ma, and Dr. Edmond Cretu in my dissertation committee.

I would like to thank my colleague Mr. David Plackett from Dr. Burt's lab, Hatef Rahmani, and Farzad Foroughi from Dr. Green's lab and my fellow lab mates, Dr. Farzad Khademolhosseini, Aurora Chen, Zhengmu Wang, Kaiwen Yuan for their insightful comments and help.

Finally, I would love to thank my supportive parents who always have been a source of inspiration and motivation in my life and I owe them the greatest degree of appreciation. 
Dedication

Ta. lly OPronts

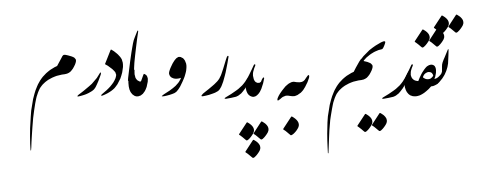




\section{Chapter 1: Introduction}

\subsection{Drug Delivery}

Despite tremendous improvements in medicine, their usage is restricted due to the associated and inevitable severe side effects. Common treatments involve direct administration, leading the drug to spread through the whole body. This impacts other healthy organs while only partial of it reaches the defected zone. Not only does this drastically decrease the treatment efficacy, but also highly increases the potency of damaging or even failure of healthful organs. On the other hand, new genetically engineered medicines also call for new methods of delivery [1]. Furthermore, investing on improving delivery techniques are more cost effective than amending the pharmaceutical characteristics of drugs [1]. To tackle these issues, drug delivery has emerged that aims toward releasing the drug exactly at the point where the source of disease exists.

However, this notion at first started with tablets and capsules which are currently known as oral drug delivery. Although, this method has been established over 1000 years ago by using coated pills, it is still under development since it has turned out to be one of the most convenient and reliable approaches [2]. Numerous drug delivery methods have been introduced based on different perspectives and purposes such as, site of action, type of drug carrier, route of delivery, and so on. These methods encompass polymeric drug delivery, zero order drug delivery (i.e: osmosis approach), nanoparticles, and microelectromechanical (MEMS) based delivery systems. Among them, the latter group is mostly associated to the advanced concurrent generation of drug delivery era which focuses on addressing both physicochemical and biological barriers [3]. 


\subsection{Controlled Drug Release System}

The most significant characteristics of today's drug delivery systems are controllability and adjustable release to maintain drug concentration in blood. As illustrated in Figure 1, the permitted drug concentration in blood is restricted by two factors. The upper limit is defined as the maximum safe concentration (MSF) above which drug becomes toxic, and the lower limit is defined as the minimum effective concentration (MEC) below which the drug is ineffective. The red-dotted curve corresponds to conventional drug delivery, which is carried out either by direct administration using syringe or taking tablets. As shown in Figure 1, this method has no control on the drug dosage, and higher drug doses must be injected in order to compensate for the dissipation of the therapeutic agent. However, this results in an initial dose burst which causes the drug to be destructive for some period. The medicine loses its productivity and its concentration drops to less than the minimum prescribed dose. On the other side, the blue plot represents a controllable drug delivery system capable of maintaining the drug concentration within the therapeutic window.

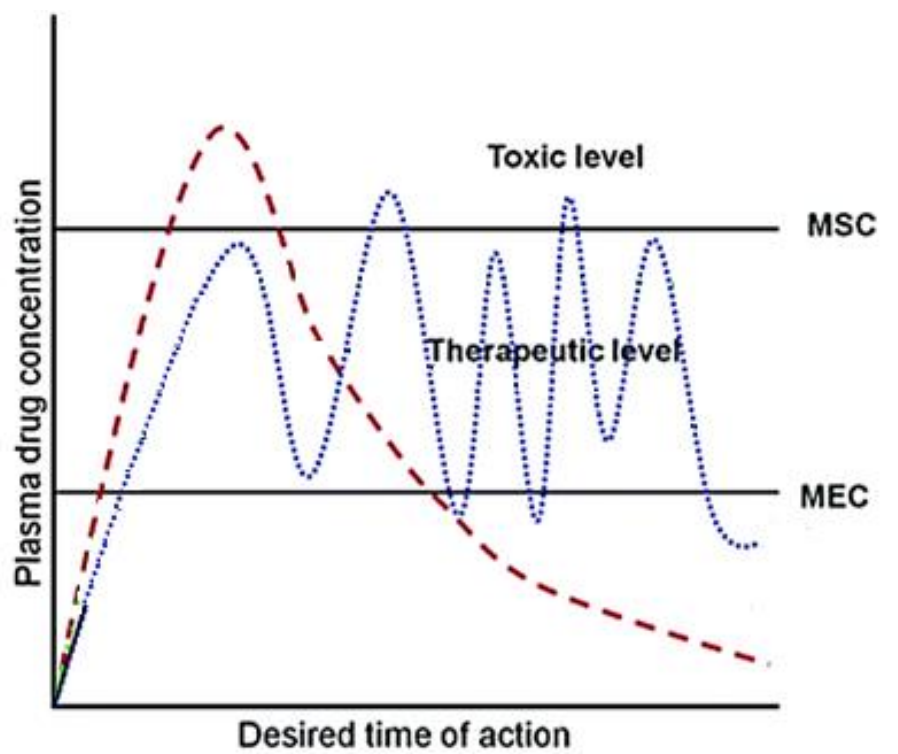

Figure 1- Function of different types of drug delivery according to therapeutic window [10] 
Another imperative aspect is adjustability of drug dose introduced by a drug delivery agent. Although some of the proposed devices have shown successful controllable release, they cannot provide that much flexibility in altering the release rate or changing the amount of the injected drug. Patient condition is a dynamic situation which can change any time and might require different treatment protocols. Thus, the drug delivery device has to adapt to the new condition by tuning the amount of introduced drug to achieve the required concentration.

\subsubsection{Polymeric drug delivery}

Polymers as drug carriers have been developed over sixty years ago, where the drug is embedded within a polymer matrix and is further released in the body. This approach has appreciable advantages of maintaining plasma drug concentration in the blood stream [4], promoting solubility of drugs [5], and enhancing the pharmacokinetic behaviour of drugs [6]. Although polymer-drug conjugates are mostly associated with zero-order drug delivery, there have been efforts to improve the controllability by incorporating technics such as $\mathrm{pH}$ sensitive polymer [7] and temperature triggered polymer [8]. Even though these techniques, in addition to other methods such as ultrasound, electromagnetic radiation, light, and so on, have led to disciplined discharged medicine [9], injecting on-demand exact doses of the drug or altering the release rate are challenges in this approach.

\subsubsection{Reservoir based device}

Another perspective in the drug delivery field which has attracted attention is reservoir based drug delivery. In this method, micro devices are fabricated into which a reservoir is designated 
for the drug payload. The outmost important factor in this approach is the method of stimuli such as osmosis, electrical, magnetic, etc, which is responsible for the drug administration.

\subsubsection{Osmosis based method}

Osmosis as a chemical phenomenon relies on the concentration gradient and provides a safe way of actuation which is able to push the drug out of the reservoir. This approach has been implemented both in oral drug deliveries and as implantable micro pumps [10]. In oral delivery, tablets with semipermeable membrane is usually employed with a hard core where the drug is regularly situated, and an aperture is provided for the drug ejection [11], [12]. Similarly, implantable micro pump devices have been manufactured emulating the same concept as above [13], [14]. In both cases, water diffusion through the tablet or micro pump causes the drug to be released from the allocated repository. This method functions easily, requires no power, includes elevated robust design of pump [15], in addition to inexpensive and large scale production [16], [17]. However, to date and to our knowledge, since this approach is passive delivery of drug, dynamic precise dosage control and pulsatile release rate have not been achieved yet.

\subsubsection{Electrical stimuli}

Electrical stimulus offers another practical way of triggering drug injection. Several microfabricated devices have been presented where a micro reservoir consisting of the drug solution is sealed with a thin metallic electrode [18], [19]. A predetermined applied current leads to the dissolution of the top electrode, leading to eventual exposure of the reservoir's content. However, a modified version of this method utilized another electrode at the bottom of the well to produce bubbles to push the drug out of the reservoir [20]. Although this method requires low 
power to operate, this dependency is nevertheless a disadvantage. Other methods have been proposed based on hydrogel, where drug delivery occurs by applying an electrical field through the hydrogel matrix [21]. The electrical field either forces the entrapped ionized drug particles to expel [22] or leads the hydrogel to deswell and liberate the drug [23]. Even though pulsatile ondemand release has been attained in recently proposed methods, power and wiring connections act as limiting factors which restrict their usage, especially when it comes to implantation.

\subsubsection{Magnetic stimuli}

Among various triggering methods, magnetic actuation has shown better compliance with expectations and requirements by providing safe, remote, powerless, and tuneable stimulus. In addition to convenient implementation by employing a simple permanent magnet for actuation. Despite broad investigations conducted on magnetic nanoparticles used for targeted drug delivery [24], [25], recently implantable devices incorporated with magnetic elements has attracted attentions.

Cai et al. used $\mathrm{Fe}_{3} \mathrm{O}_{4}$ particles inside the drug reservoir to obstruct the pores of the permeable membrane covering the reservoir, controlling the drug diffusion [26]. However, this device, requires a reversible magnetic field for both on/off mode of the drug diffusion. Rahimi et al. employed a thermosensitive hydrogel triggered by a resonant heater which was remotely actuated with a determined frequency of the applied magnetic field [27]. This hydrogel served as a valve, therefore, the loaded drug was exposed and diffused to the environment when it was opened. However, temperature rise may be problematic for both body and the device operation. Ferrogels are another promising substance suitable for drug delivery purpose. Studies on the fundamental components engaged in ferrogel formation has proven the capability of this material 
to be used as a controlled drug delivery agent [28]. Zhao et al. reported a highly permeable ferrogel fabricated for pulsatile on-demand cell and drug delivery [29]. However, apart from the difficulties in fabrication process such as sensitive temperature conditions, incessant drug diffusion from ferrogel has remained an issue.

Pirmoradi et al. created a magnetic membrane which covered the drug reservoir and was deflected whenever a magnetic field was applied [30], [31]. Inward deflection of the magnetic membrane caused the drug solution to be pumped out of the reservoir through the provided micro aperture on the membrane. This concept has been improved for the treatment of prostate cancer by using a separated magnetic block attached to the membrane [32]. Instead of having the magnetic membrane, the magnetic block is responsible for the membrane deflection when an external magnetic field is applied. Two different versions of this device have been fabricated: a PDMS device which is the larger device and the 3D-printed one which is small enough to be implanted by a needle. However, the biocompatibility of the 3D-printed device is a concern.

Porous structures made by $\mathrm{Fe}_{3} \mathrm{O}_{4}$ has shown promising adsorption and release of methyl blue as a model drug [33]. Although this magnetic porous surface could not release the methyl blue in a DC magnetic field, oscillating magnetic fields demonstrated considerable release. Nevertheless, combining DC and AC field resulted in better release as it is believed that magnetic particles are aligned in a DC filed while AS filed leads to oscillation, thus drug discharge occurs. The $\mathrm{Fe}_{3} \mathrm{O}_{4}$ structure has been reported to be biocompatible, but this porous material has limited capacity in absorbing the drug.

Hoare et al. has employed a thermosensitive composite membrane covering the drug reservoir [34]. This membrane was fabricated by embedding the engineered super paramagnetic magnetite nanoparticles along with thermosensitive nanogels into the ethyl cellulose which was utilized as 
the base of the membrane. Upon exposing this membrane to an oscillating magnetic field, vibration of the magnetite nanoparticles induce heat causing the entrapped nanogels to shrink. This will cause the drug to diffuse through the temporary created cavity. By removing the magnetic field, the device cools down leading the nanogels to reswell and retain their initial condition. Therefore, the cavity will be closed and drug diffusion terminates.

The following table summarizes the proposed reservoir based drug delivery methods and highlights their features.

Table 1- Summarized reservoir based drug delivery methods

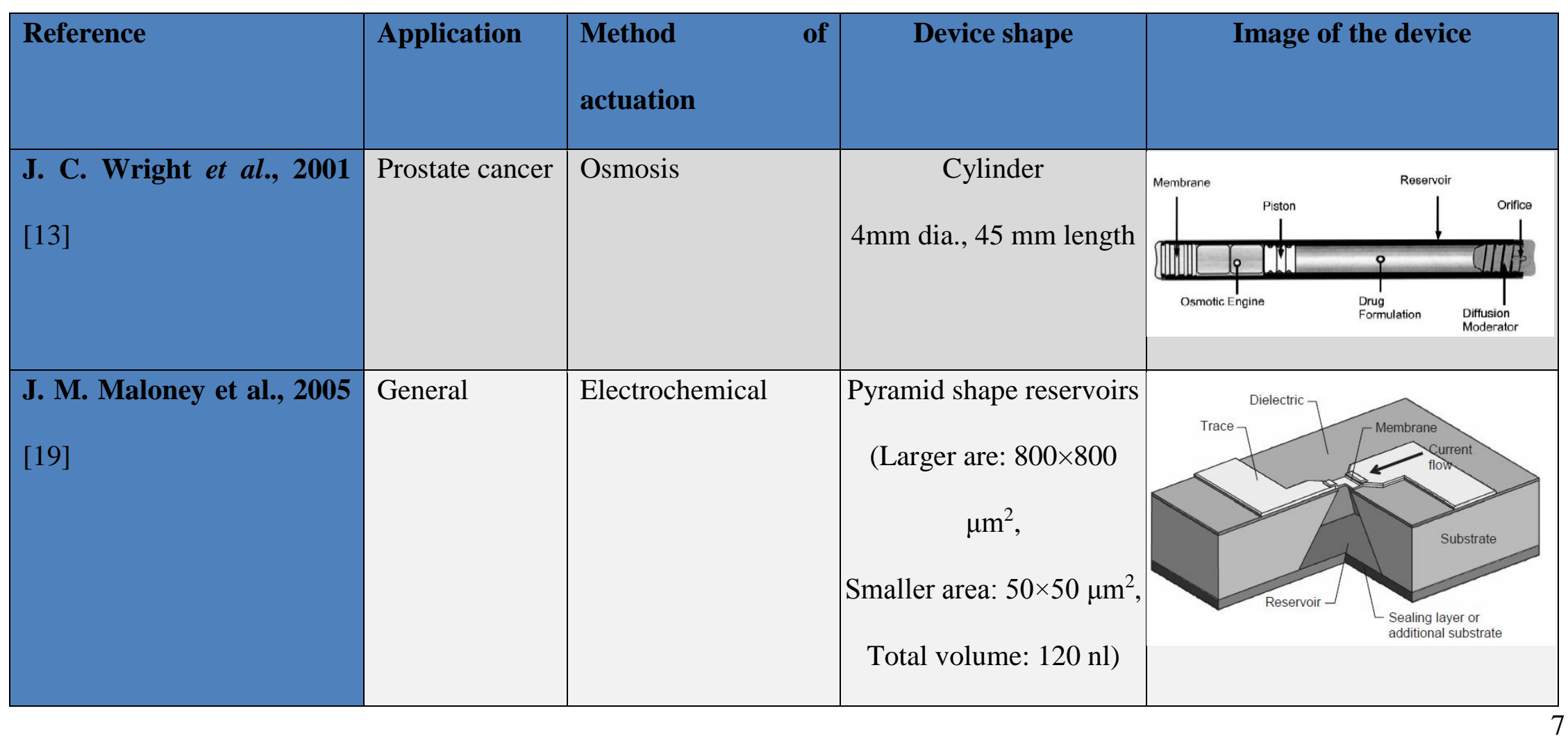




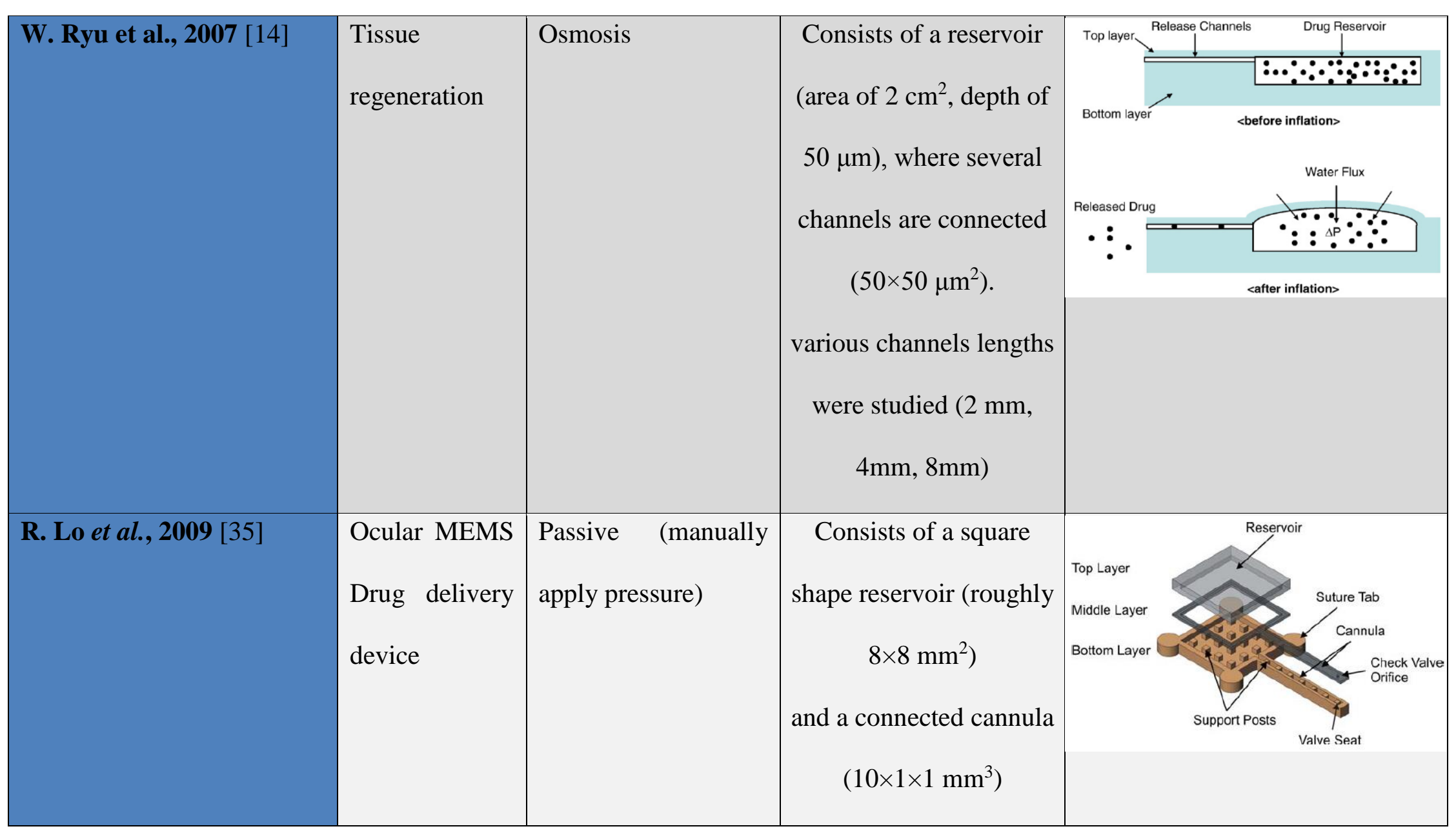




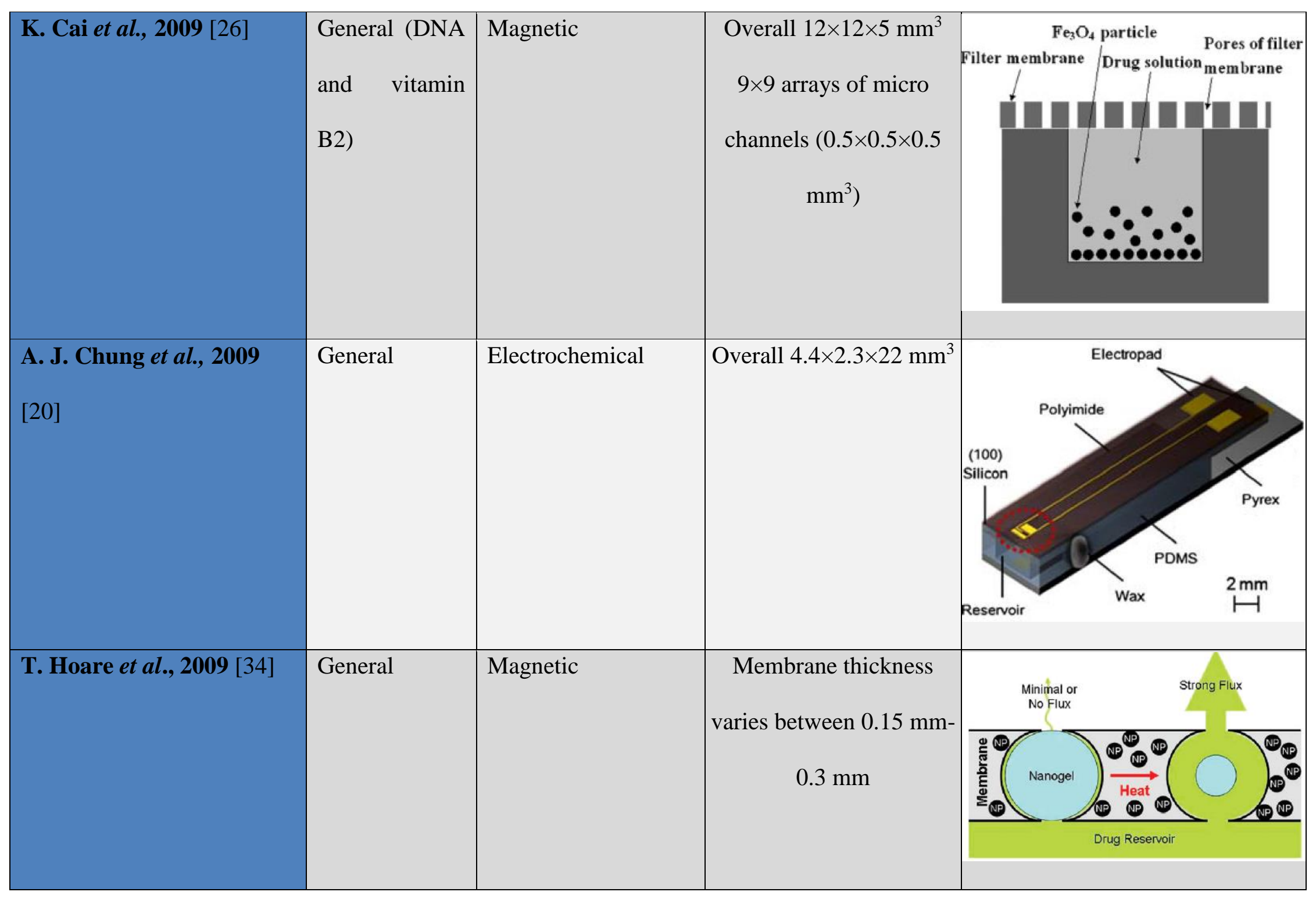




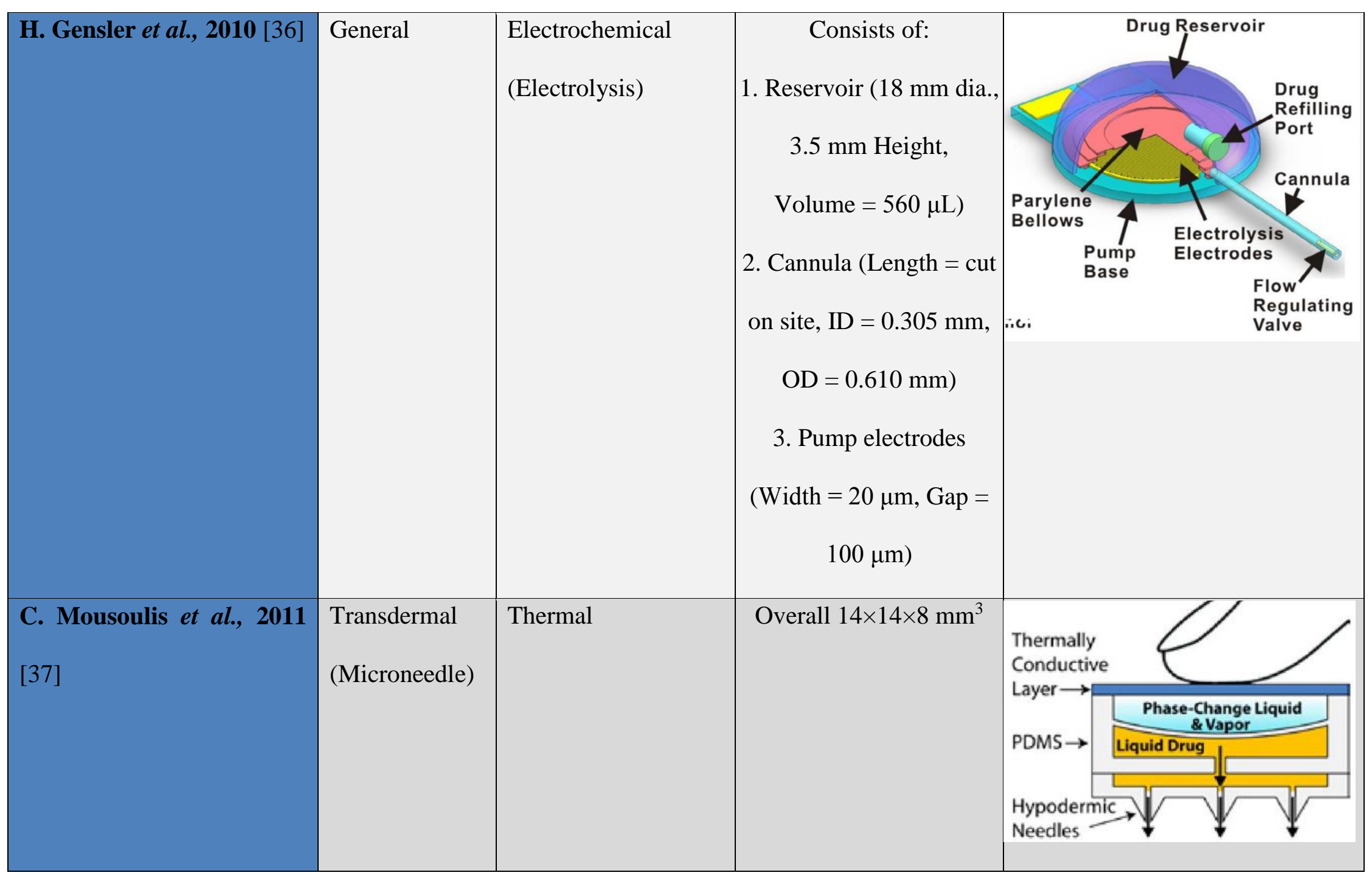




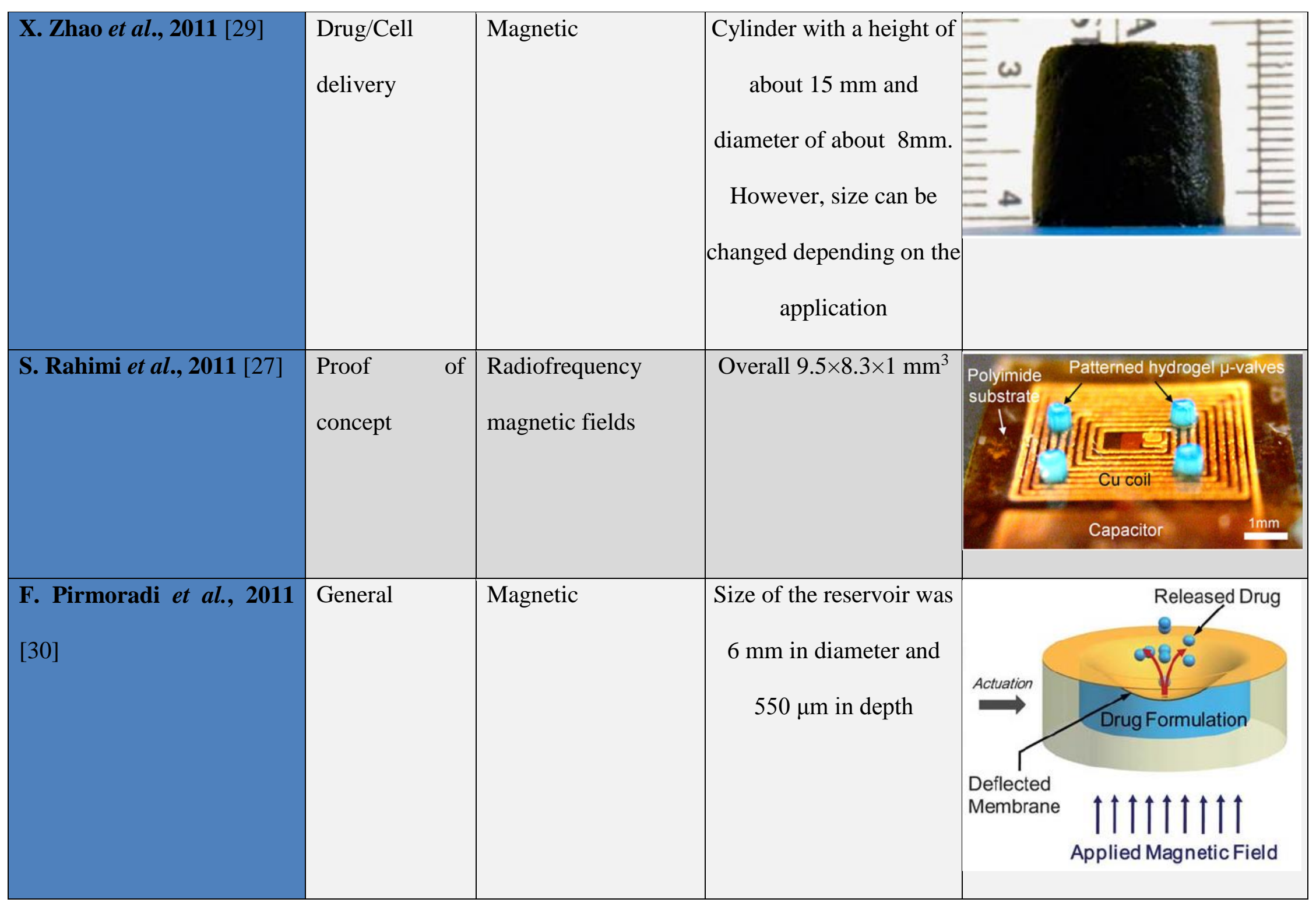




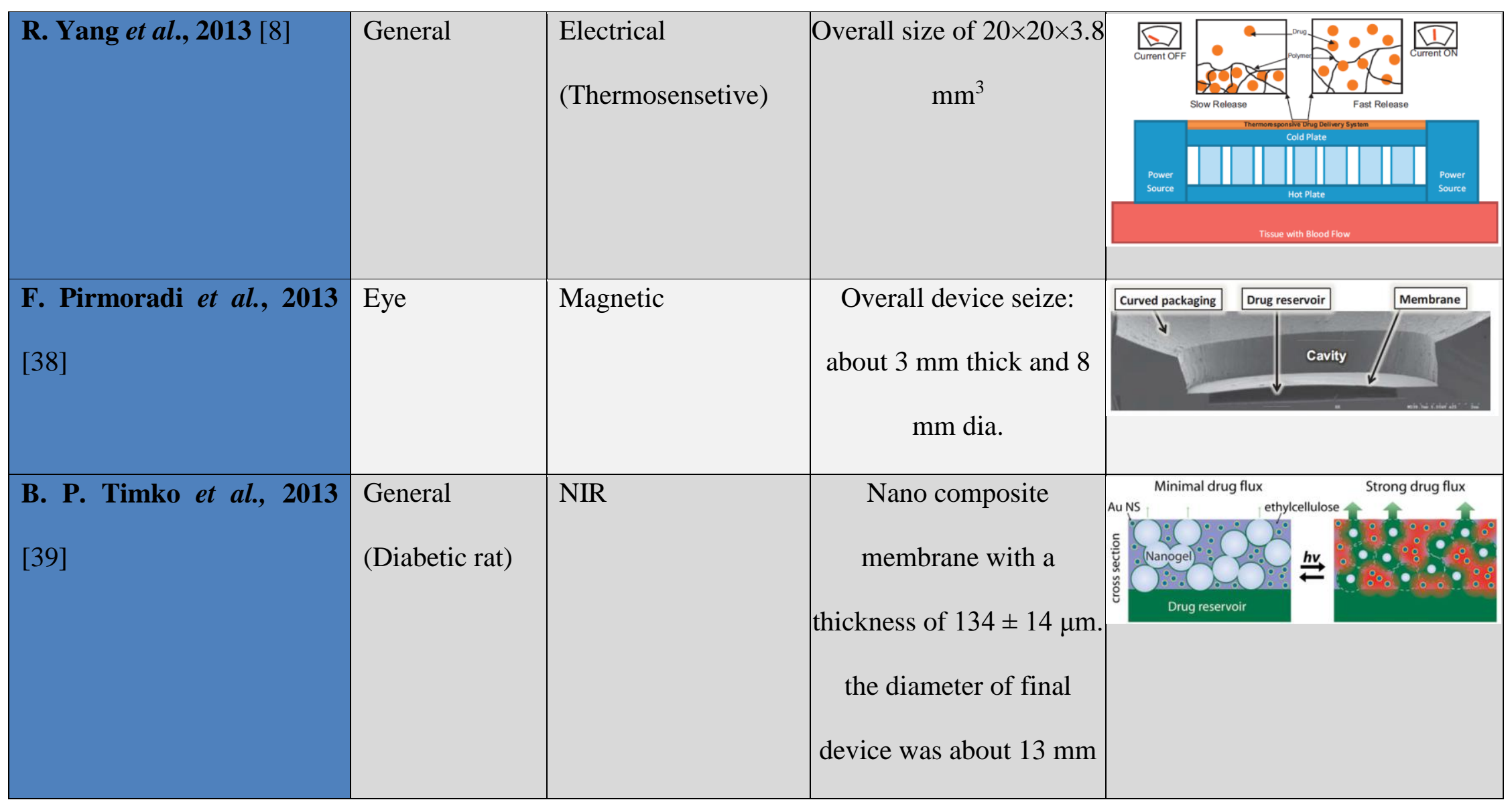




\begin{tabular}{|c|c|c|c|c|}
\hline 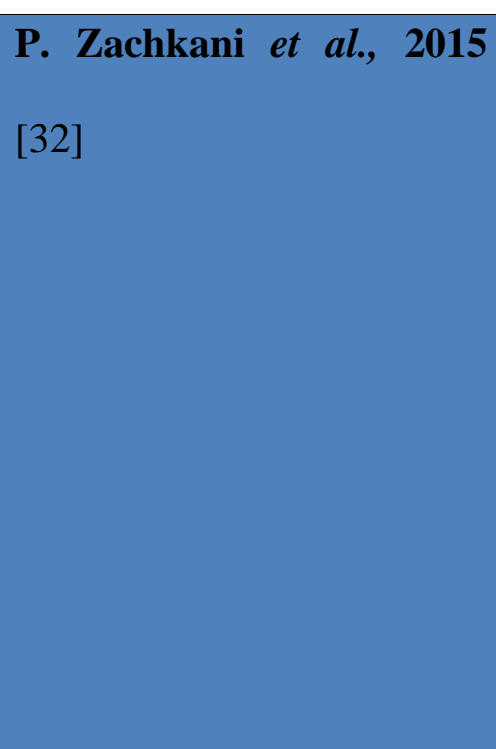 & $\begin{array}{l}\text { Prostate } \\
\text { Cancer }\end{array}$ & Magnetic & $\begin{array}{c}\text { Larger device overall } \\
\text { dimension: } \\
(\mathrm{OD}=5 \mathrm{~mm}, \mathrm{ID}=3 \mathrm{~mm}, \\
\text { length }=12 \mathrm{~mm}) \\
\text { Smaller device overall } \\
\text { dimensions: } \\
(\mathrm{OD}=2 \mathrm{~mm}, \mathrm{ID}=1 \mathrm{~mm}, \\
\text { length }=12 \mathrm{~mm})\end{array}$ & Bousing Membrane \\
\hline Y. T. Yi et al., 2015 [22] & General & Electrical & $\begin{array}{l}\text { Device has a reservoir } \\
\text { with } 3 \mathrm{~mm} \text { dimeter and } \\
1.5 \mathrm{~mm} \text { length, and An } \\
\text { aperture with } 0.5 \mathrm{~mm} \\
\text { diameter and } 0.2 \mathrm{~mm} \\
\text { height }\end{array}$ & Bop electrode \\
\hline
\end{tabular}




\subsection{Current Challenges}

As discussed in previous sections, various drug delivery systems have been proposed based on different applications and method of release. However, there are serious issues when it comes to implantation. Biocompatibility, size limitation, and long term functionality are common issues, most of which have been well addressed. Nowadays, the critical barriers are release rate, manipulation and drug dosage control. In this regard, active drug delivery systems play a vital role. Although some proposed devices are able to control the drug release, they rely on drug diffusion. Hence, they cannot alter the amount of introduced drug and change the drug dose. The type of stimuli is another crucial factor affecting device performance. Electrical stimulus demands power and wire connections, limiting their application for implantable devices. To obviate the need for power, advanced methods using smart material such as near infrared (NIR) devices [39], temperature based systems [8], and ultrasound triggered approach [40] have been made. Nevertheless, when the device is implanted, it is no longer accessible and other parameters such as body temperature, biofilm formation, and hormone release may interfere with device performance. Magnetic actuation requires no power, is doable remotely, and has no side effect or danger for patients.

To summarize, according to the literatures, it is deduced that a successful drug delivery device has to be biocompatible, implantable and release the drug on demand. It is also expected to be able to alter the release rate and drug dosage whenever it is necessary. Additionally, the method should be efficiently functional for a long term treatment. 


\subsection{Magnetic Sponge as an On-Demand Drug Delivery Device}

In this thesis, a biocompatible drug delivery device has been developed based on a magnetic sponge which is shrunk whenever a magnetic field is applied, resulting in releasing its contents. The amount of shrinkage is dependent on the magnetic properties of the sponge and proportional to the strength of the applied magnetic field. The base material used in fabrication of the sponge is polydimethylsiloxane (PDMS), which is mixed with carbonyl iron microparticles to obtain magnetic PDMS. This magnetic PDMS is further rendered porous by utilizing a solvent casting and particulate leaching technique. Although this sponge can solely be used as a drug delivery agent, a separated reservoir has been designed and fabricated in order to enhance the controllability and decrease the leakage.

Overall, this device has $6 \mathrm{~mm}$ diameter and less than $2 \mathrm{~mm}$ thickness. Device release performance has been tested using methylene blue and docetaxel as a surrogate and real drug, respectively. This device was placed inside the porcine bladder tissue and actuated several times to assess device performance while it is implanted. Figure 2 shows schematic exploded view of the device components including, reservoir, magnetic sponge, and membrane.

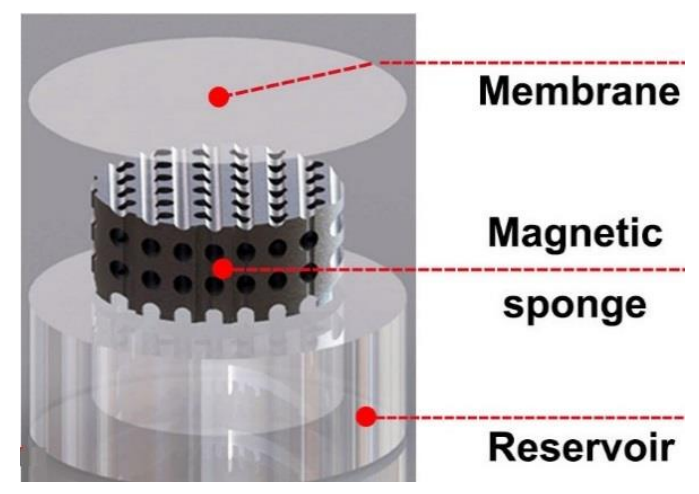

Figure 2- schematic view of the device components

This device requires no power, can release on-demand, and can adjust the amount of injected drug by either tuning the magnetic field or increasing the number of actuation. The release may 
be initiated by a piece of strong magnet (e.g. N52 neodymium magnet). The operational magnetic field strength is much higher than the magnetic field caused by common electrical devices such as cell phone, laptop, or etc., thus, no interference may occur.

\subsection{Thesis Overview}

This thesis has been presented with an introductory chapter followed by two chapters and a conclusion which summarizes the efforts and suggests future steps to develop this project.

In the first chapter, literature has been reviewed within the scope of implantable proposed drug delivery devices. Different types of drug delivery systems including polymer based, and various well established methods of actuation have been introduced. Existing deficiencies and persisting drawbacks have been studied, based on which this thesis objective has been defined.

Chapter two focuses on design and fabrication processes of the magnetic sponge and the final proposed drug delivery device. Firstly, the fabrication steps of magnetic porous PDMS are demonstrated and later its features such as mechanical properties are investigated. After obtaining the best case for a magnetic sponge, fabrication of the reservoir and membrane are described. Eventually, the final device is presented by assembling the components. Once the device fabrication is covered, further experiments were performed to evaluate device performance.

Chapter three covers the results obtained from the experiments. This begins with data acquired from testing the functionality of the magnetic porous PDMS in various magnetic fields, which ends up with finding the best case for drug delivery. Later, device evaluation is carried out by interpreting the data attained from cumulative methylene blue release and docetaxel release test. 
Implantability of the device is investigated by inspecting injection behaviour in porcine bladder tissue.

Chapter four summarizes the project carried out in this thesis including general conclusions and elucidates possible future attempts which may result in development of the proposed drug delivery method. 


\section{Chapter 2: Development and Characterisation of the Magnetic Porous PDMS Drug Delivery Device}

\subsection{Porous PDMS}

Different methods have been presented for porous polydimethylsiloxane (PDMS) fabrication. However, most of them are based on a solvent casting and particulate leaching (SCPL) technique [41]-[44]. The aim of this technique is to introduce solid particles within the structure of PDMS and extract them after curing PDMS. Vacant spaces are created in the polymer matrix which makes it porous. As declared, this technique has two leading parameters: particle selection and method of extraction. Although, some suggested $\mathrm{NaCl}$ as the solute agent [43], [44], and later removed it by dissolving the salt, a much easier approach is proposed where similar results are obtained in a fast and convenient way.

\subsection{Magnetic Porous PDMS}

To make a magnetic porous PDMS, two separated tasks should be carried out. One task focuses on the porous scaffold and the other one is responsible for magnetic PDMS. Magnetic porous PDMS is created by merging these two items.

\subsubsection{Task 1: preparing porous scaffold}

As mentioned earlier, sugar is used in our method as the soluble particle. The following steps indicate the fabrication process of porous scaffold: 
Step 1: $20 \mathrm{ml}$ of sugar was mixed with about $0.5 \mathrm{ml}$ of water, and then poured into a small size Petri dish.

Step 2: By pressing the sugar lump firmly and uniformly, sugar particles' contact is ensured. Controlling the amount of sugar and final volume of the lump play crucial roles in determining mechanical properties of the subsequent magnetic porous PDMS.

Step 3: the existing moisture was evaporated by placing the Petri dish in a convection oven for about 15 minutes. This helps connected sugar particles structure to be formed.

\subsubsection{Task 2: Magnetic PDMS}

Step 1: First of all, PDMS (Sylgard 182 Silicone Elastomer, Dow Corning Corporation) was made with 1:30 cross-linker to base proportion.

Step 2: In order to induce the magnetic properties, Carbonyl Iron (CI) ferromagnetic microparticles were added into the PDMS. Concentration of the CI particles dictates the quality of magnetic properties of the PDMS. Thus, three different CI to PDMS w/w ratio has been made $(50 \%, 100 \%$, and $150 \%)$ to find the best case (the criteria led to find the best concentration is explained later on). In order to achieve better dispersion of CI microparticles in PDMS matrix, about $1 \mathrm{~mL}$ of Isopropanol (IPA) was dispensed during the mixing process which later evaporated. IPA also makes the mixture less viscous, facilitating easier stirring and dispersion. The mixing process takes about ten minutes.

\subsubsection{Magnetic Porous PDMS}

Once the porous scaffold and magnetic PDMS are prepared, the magnetic sponge can be made by adding magnetic PDMS to the sugar lump which occurs as the following steps: 
Step 1: The magnetic PDMS was poured on the porous scaffold made out of sugar. The magnetic PDMS penetrates through the pores of the sugar lump until it encompasses the whole structure and reaches the bottom of the Petri dish. To expedite the process, this step was performed inside the vacuum chamber. Using a vacuum chamber is also required to dissipate the PDMS.

Step 2: Magnetic PDMS was cured in an oven at $70^{\circ} \mathrm{C}$ for 3 hours to form the magnetic porous PDMS scaffold.

Step 3: Eventually, several samples were cut from the scaffold and immersed in water for 2-4 hours to dissolve the sugar. Warm water and stirring may be utilized to accelerate this step. Dissolved sugar particles leads to vacant spaces which form the pores of the sponge. Besides, the chain connection of sugar particles results in interconnected pores. Figure 3 shows the magnetic PDMS scaffold out of which one sample was cut, with the final magnetic porous PDMS dimensions of $6 \mathrm{~mm}$ diameter and $5 \mathrm{~mm}$ height.

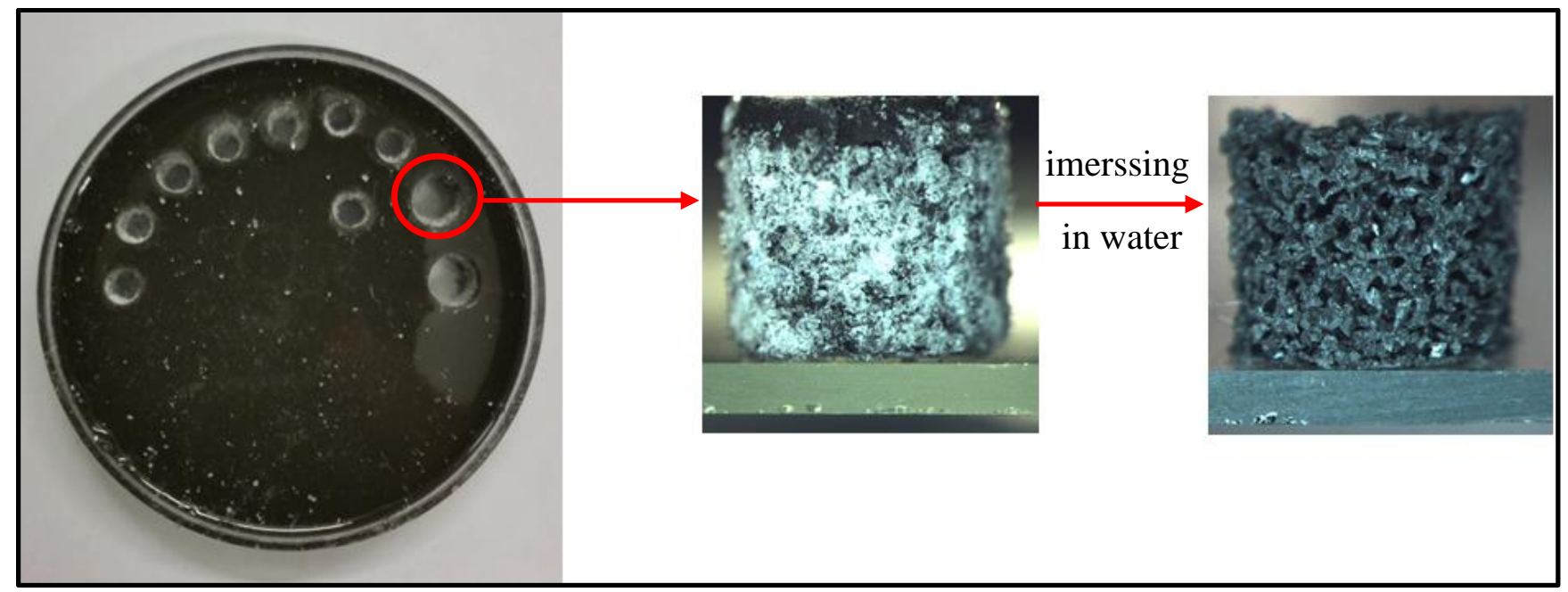

Figure 3- From right to left; magnetic porous PDMS scaffold, sample punched from the scaffold, and the consequent magnetic sponge after submerging the sample in water 


\subsection{Characterization of Magnetic Porous PDMS}

The magnetic sponge has two crucial elements: magnetic properties and porosity. Both of these directly impact its mechanical properties and shrinkage. As declared previously, magnetic porous PDMS has been made with three different carbonyl iron concentrations. In this section, magnetic sponge behavior in various magnetic fields is studied with the intent of finding the best CI to PDMS w/w ratio.

\subsubsection{Porosity}

Porosity is defined as the ratio of the vacant space volume to the total volume of material. Although, the pore's configuration is generated through a random process, porosity is the main influential parameter which can tailor the mechanical properties of a porous substance. The porosity of the sponge is dependent on the sugar structure and can be altered either by changing the amount of the sugar and/or the sugar lump compression.

To evaluate the porosity of the sponge made according to the aforementioned fabrication steps, three different samples were selected randomly from three different scaffolds fabricated separately and independently. Each sample was then immersed in water and placed in a vacuum so they were filled with water. As the density of the sponge is less than water, a magnet may be used to submerge the samples deep into water. The shrinkage caused by the magnet assists in venting the air inside the sponge. After about 20 minutes, samples were removed and weighed. By subtracting the weight of sponge before and after submerging, the amount of absorbed water is calculated. The weight of the water entrapped in pores of the sponge is equivalent to the volume of the total pores of the sponge. Hence, the obtained porosity was $0.625 \pm 0.022$ with the low deviation (less than 3.5\%) verifiying the consistency of sponges in fabrication. 


\subsubsection{Carbonyl Iron Concentration}

A cross-section view of the magnetic sponge including SEM images from samples with different concentrations are provided in Figure 4. White dots represent the CI micro particles which are more evident as the concentration soars. Increasing the concentration of CI ferromagnetic particles improves the magnetic properties of the sponge and consequently raises the magnetic force exerted on the sponge in a magnetic field. However, the main objective is to achieve as much shrinkage as possible in various magnetic fields since it corresponds to more drug release. Accordingly, in order to characterize the influence of the CI concentration on the sponge deformation, a test has been conducted in which the amount of sponge displacement was measured versus different magnetic fields strength. Samples with different concentration were chosen with the roughly same size $(5 \mathrm{~mm}$ in diameter and about $6.5 \mathrm{~mm}$ in length) and exposed to different magnetic field strengths.

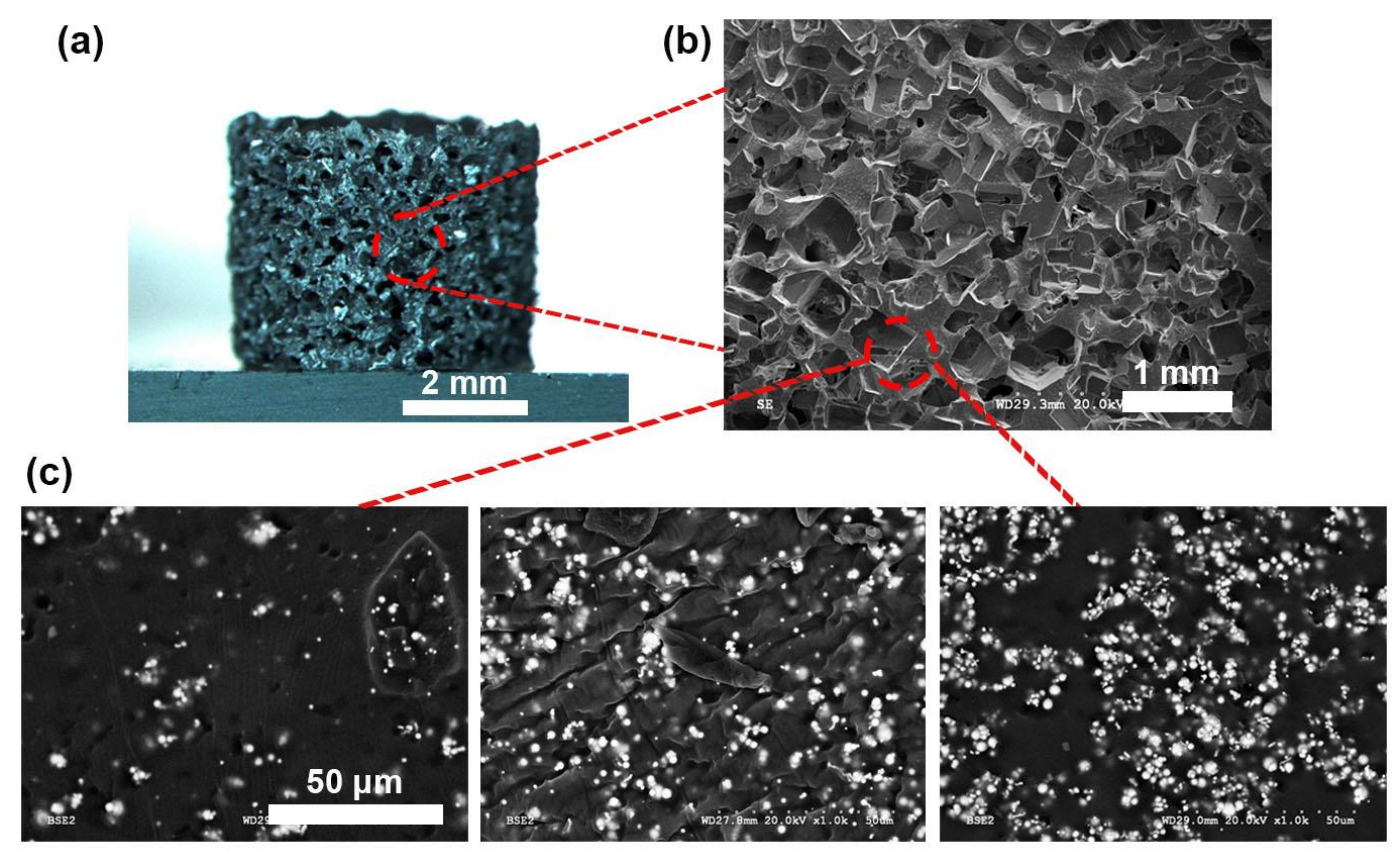

Figure 4- a) Sponge, b) Cross section of the magnetic porous PDMS, c) Different CI to PDMS wt\% ratios, from left to right respectively, 50\%, $100 \%$, and $150 \%$ 
As depicted in Figure 5, although the sample with the ratio of $100 \%$ was squeezed more than $50 \%$ one, $150 \%$ ratio had less displacement than $100 \%$ as well. In fact, another facet of introducing microparticles into the PDMS matrix is the elevation of the elasticity modulus. In this regard, adding carbonyl iron particles will increase the elasticity modulus of PDMS considerably, especially in high concentrations (more than 100\%) [45]. Thus, this parameter acts as a resistive factor opposing the magnetic force applied to the sponge. As a proof to this justification, the elasticity modulus of all samples with different ratios including a porous PDMS have been measured using thermo-mechanical analyzer (TMA 2940-Q series, TA, Instruments, DE, USA).

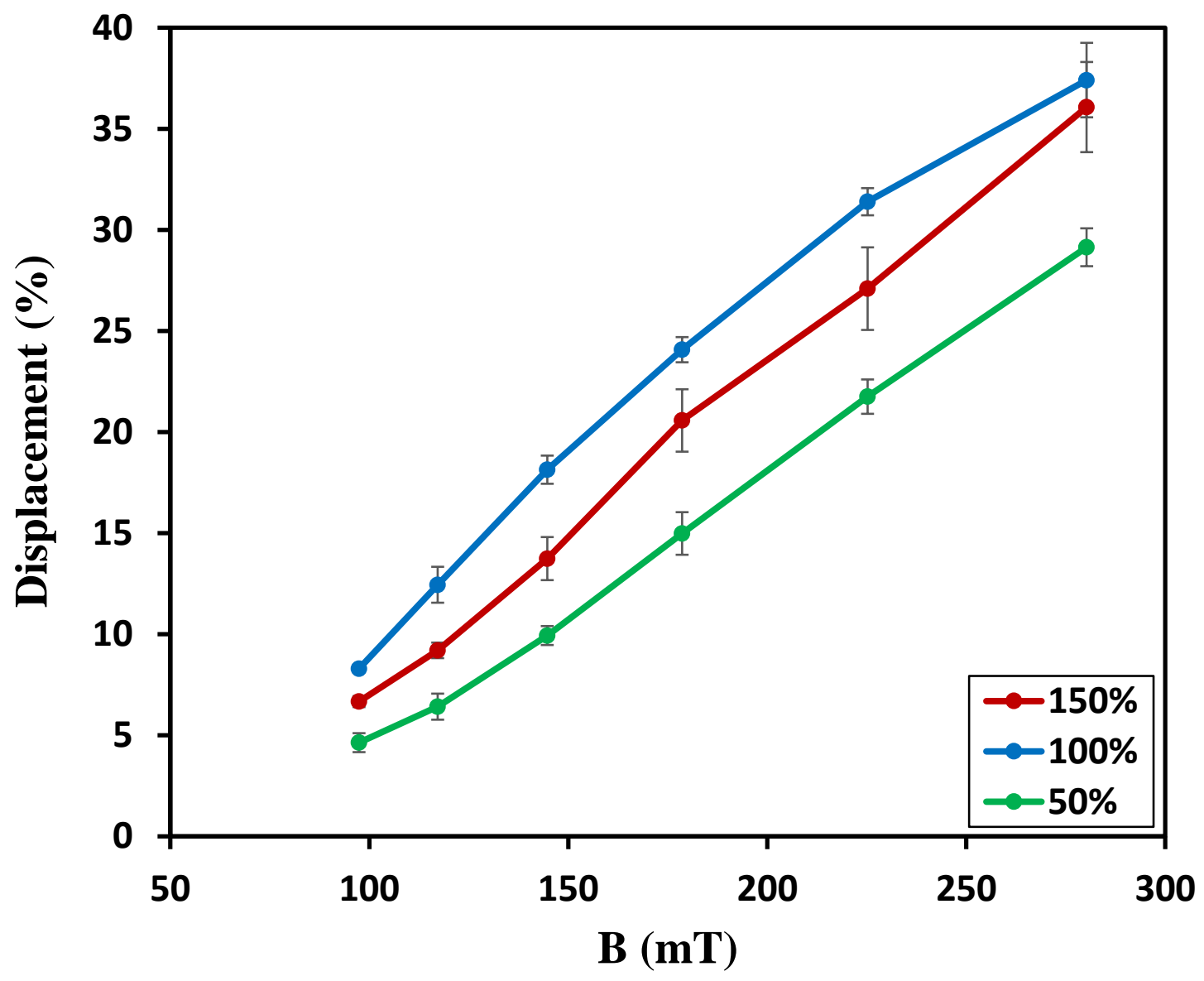

Figure 5- Displacement of sponges with different CI concentration in various magnetic fields 
The result has been plotted in Figure 6, which exhibits increase in elasticity modulus as the concentration of the CI particles raise up. However, from $100 \%$ to $150 \%$ ratio, this increment is much higher than the gap between $50 \%$ and $100 \%$ ratios or even generally among preceding concentrations. Similar trends were observed and reported by Li et al. [45]. Hence, for the drug delivery device purpose, $100 \%$ ratio has been selected. The acquired elasticity modulus are 4.56 $\mathrm{kPa}, 4.76 \mathrm{kPa}, 5.03 \mathrm{kPa}$, and $6.43 \mathrm{kPa}$ for porous PDMS, and magnetic sponges with $\mathrm{CI}$ to PDMS wt $\%$ ratio of $50 \%, 100 \%$, and $150 \%$ respectively.

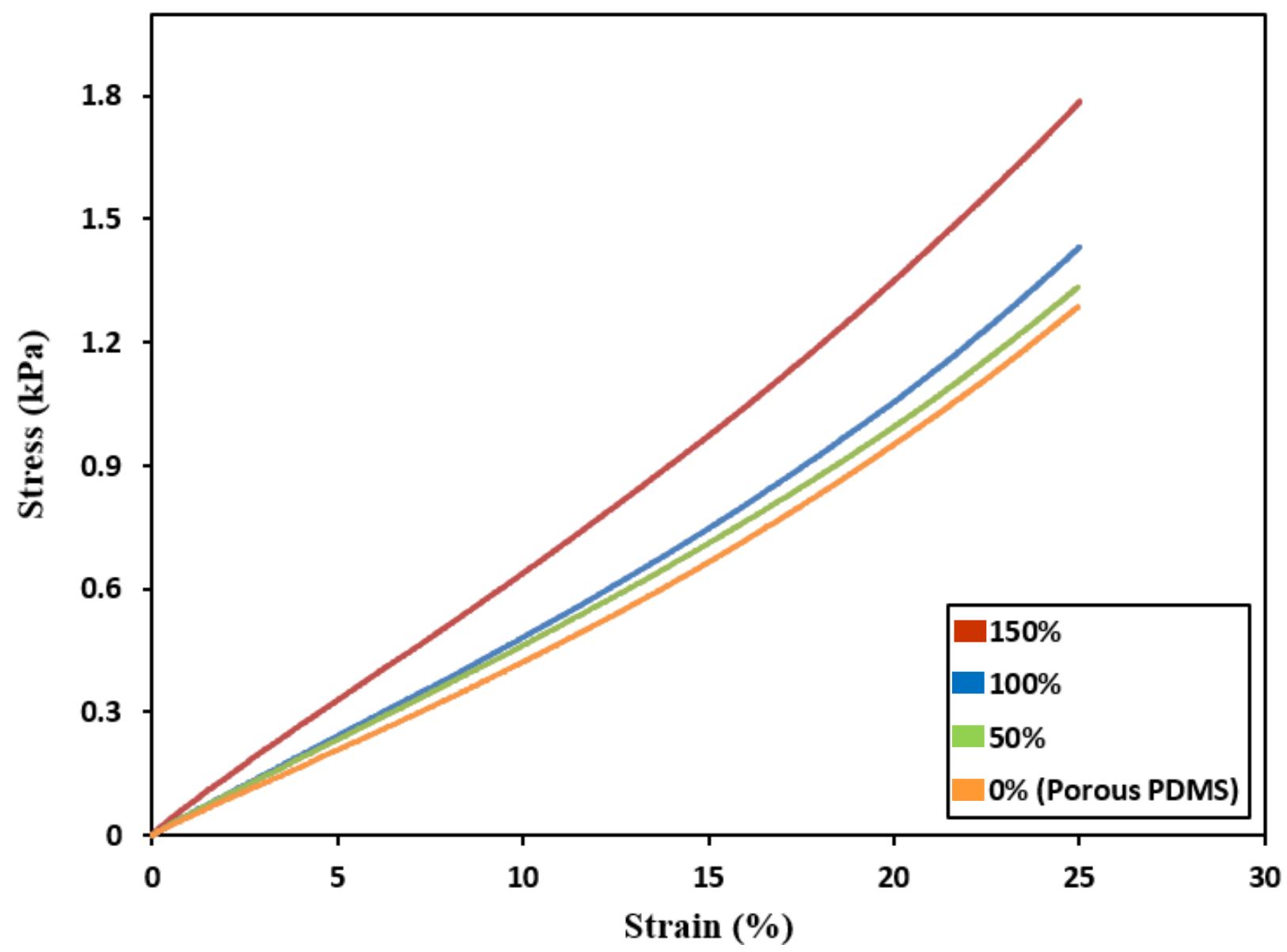

Figure 6- Stress versus strain for different sponges with 0, 50\%, 100\%, and $150 \%$ CI/PDMS wt $\%$ ratios 


\subsection{Drug Delivery Device Fabrication}

As noted earlier, this magnetic sponge could be used solely as a drug delivery agent. In this perspective, one approach is immersing the magnetic sponge in a drug solution and let it absorb the drug. Shrinkage and relaxation of the sponge in an on/off magnetic field can provide active pumping in/out of the drug solution. However, the controllability of such a system is relatively weak and the incessant release form the sponge is a major imperfection. Thus, to enhance the controllability and improve the delivery performance, a device has been designed and fabricated which encompasses the sponge. This device is composed of three main components which are as follows:

1. Reservoir: The drug is loaded inside a reservoir, where, the magnetic sponge is placed. This reservoir not only preserves the sponge, but also is able to be loaded with plenty of drug. This reservoir has depth of about $1.5 \mathrm{~mm}$ and outer diameter of $6 \mathrm{~mm}$.

2. Magnetic sponge: According to the previous section, magnetic porous PDMS with $100 \%$ CI/PDMS wt $\%$ is made and further cut to fit the reservoir. Therefore, the overall dimensions of sponge are $4 \mathrm{~mm}$ diameter and $1.5 \mathrm{~mm}$ length.

3. Membrane: A thin PDMS membrane $(10 \mu \mathrm{m})$ is designated to seal the reservoir and decrease device leakage.

Once all of these three main elements are prepared, the final device is constructed by assembling these parts. The membrane and reservoir are made from PDMS (Sylgard 184 Silicone Elastomer, Dow Corning Corporation. In the following sections, the design perspective and fabrication process of the aforementioned components are reviewed in detail. 


\subsubsection{Reservoir}

To fabricate the reservoir, a positive mold was designed in Solidworks ${ }^{\circledR}$ and built by a 3D printer (Asigo Pico, CA, USA) using Plas White a UV curable polymer. This mold, shown in Figure 7, consists of pillars with the height of $1.5 \mathrm{~mm}$ and the diameter of $4 \mathrm{~mm}$.

PDMS is made with a 1:10 cross-linker to base ratio, and poured into the mold. The PDMS was then desiccated in a vacuum chamber for 30 minutes and cured in an oven at $70{ }^{\circ} \mathrm{C}$ for 3 hours. Even though PDMS could be cured sooner in higher temperature (e.g. $200{ }^{\circ} \mathrm{C}$ ), the glass transition temperature of the $3 \mathrm{D}$ printed mold is about $83{ }^{\circ} \mathrm{C}$, which restricts the curing temperature. The PDMS layer was later demolded and reservoirs were punched with an outer diameter of $6 \mathrm{~mm}$. The final thickness of the reservoir is less than $2 \mathrm{~mm}$ (approximately $1.5 \mathrm{~mm}$ depth of the reservoir and about $0.5 \mathrm{~mm}$ base of the reservoir). However, it can be adjusted precisely by controlling the exact volume of the PDMS disposed into the mold and the height of the pillar. Although smaller reservoirs has also been made with a different mold, this particular size has been chosen based on the ocular drug delivery device dimension [38].

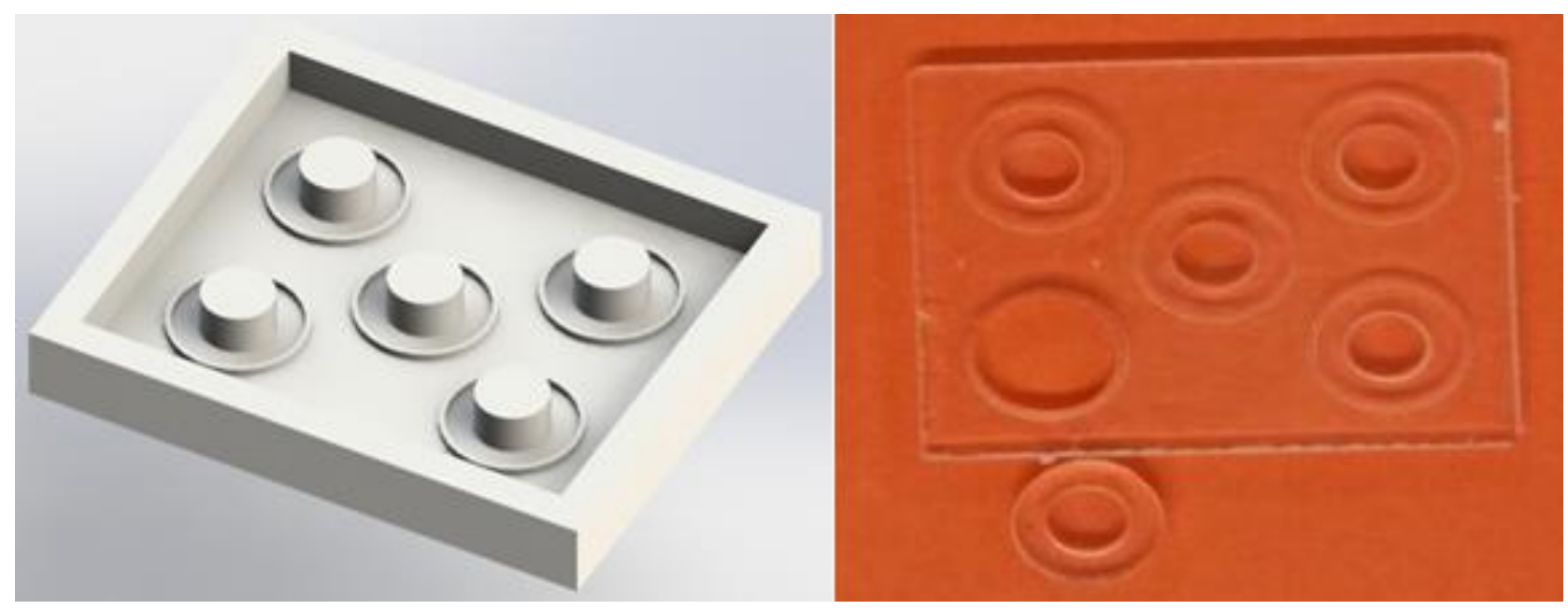

Figure 7- Mold on the left side and the demolded PDMS layer (right side) out of which one sample reservoir was punched 


\subsubsection{Membrane}

As noted above, the membrane will eventually cover the top of the reservoir after the sponge is placed. The fabrication process of the membrane is through the following steps:

Step 1: Polyacrylic acid (PAA) was prepared by mixing PAA powder $\left(\mathrm{M}_{\mathrm{w}}=1800\right.$, SigmaAldrich, ON, Canada) with distilled water to achieve a $25 \% \mathrm{w} / \mathrm{v}$ concentration. This step requires about 10 minutes vortex mixing of the solution followed by filtering, using sterile $0.45 \mu \mathrm{m}$ PVDF syringe filter (Millipore Corporation, Ma, USA).

Step 2: A pre-cleaned glass slide surface is treated by plasma to enhance surface wettability.

Step 3: The filtered PAA was spun on the glass slide in two steps $(10 \mathrm{~s}$ at $500 \mathrm{rpm}, 30 \mathrm{~s}$ at 800 rpm). The glass slide was later placed on a hot plate at $150{ }^{\circ} \mathrm{C}$ for 5 minutes to evaporate water and cooled down afterwards in 5 minutes.

Step 4: PDMS was made with 1:10 curing agent to pre-polymer ratio and degassed in a vacuum chamber for 45 minutes. The prepared PDMS was further poured on the PAA coated glass slide and spun in two steps (20 s at $500 \mathrm{rpm}, 3 \mathrm{~min}$. at $3500 \mathrm{rpm}$ ). The PDMS membrane was then cured on hot plate at $150{ }^{\circ} \mathrm{C}$ in less than 5 minutes.

Final thickness of the membrane was measured to be roughly $10 \mu \mathrm{m}$ using a surface profilometer (Wyko, VEECO Metrology Group, AZ, USA). 


\subsubsection{Assembling}

First of all, the desired amount of the drug is deposited into the reservoir. In the result and discussion section, it is illustrated why there is no concern regarding the limit of loaded drug into the reservoir. The magnetic sponge is cut to the size fitting the entire reservoir. Plasma surface treatment is applied to both the reservoir including the sponge and the membrane which enables to covalently and irreversibly bond the membrane to the reservoir. At this stage the device is completely sealed. By immersing this set into water, the sacrificial layer made by PAA is dissolved and the device is set free. Eventually, an aperture of size of $90 \times 90 \mu \mathrm{m}^{2}$ is created at the center of the membrane by laser ablation using Nd:YAG laser (Quicklaze, New Wave Research, Sunnyvale, CA). The green laser is utilized with the wavelength of $523 \mathrm{~nm}, 20$ pulses per inch (PPI), and about $10 \mu \mathrm{m} / \mathrm{s}$ scanning speed. Figure 8 shows the actual size of the device relative to one Canadian dollar. SEM images from cross section of the device is also provided in Figure 9, where the covalent bonding among reservoir, sponge, and the membrane is evident in insets $i$ and ii.

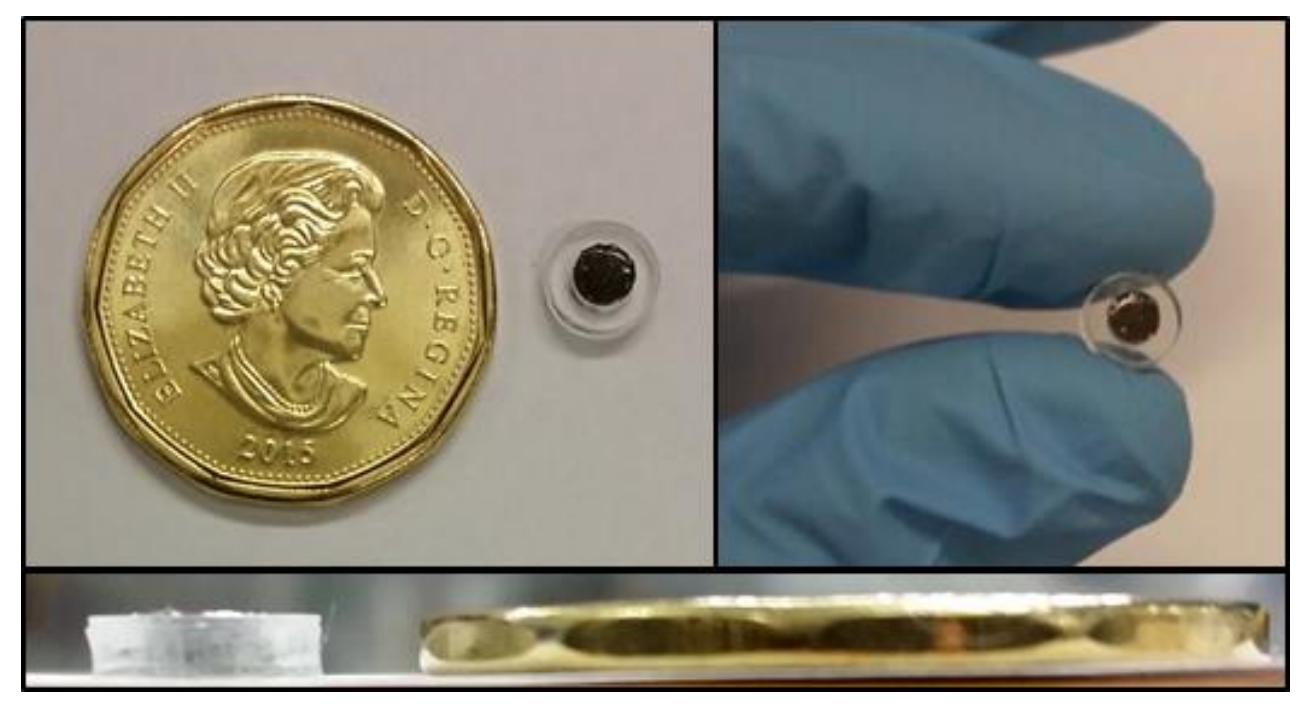

Figure 8- Real device, in comparison with one Canadian dollar. This device is also roughly as thick as a Canadian dollar 

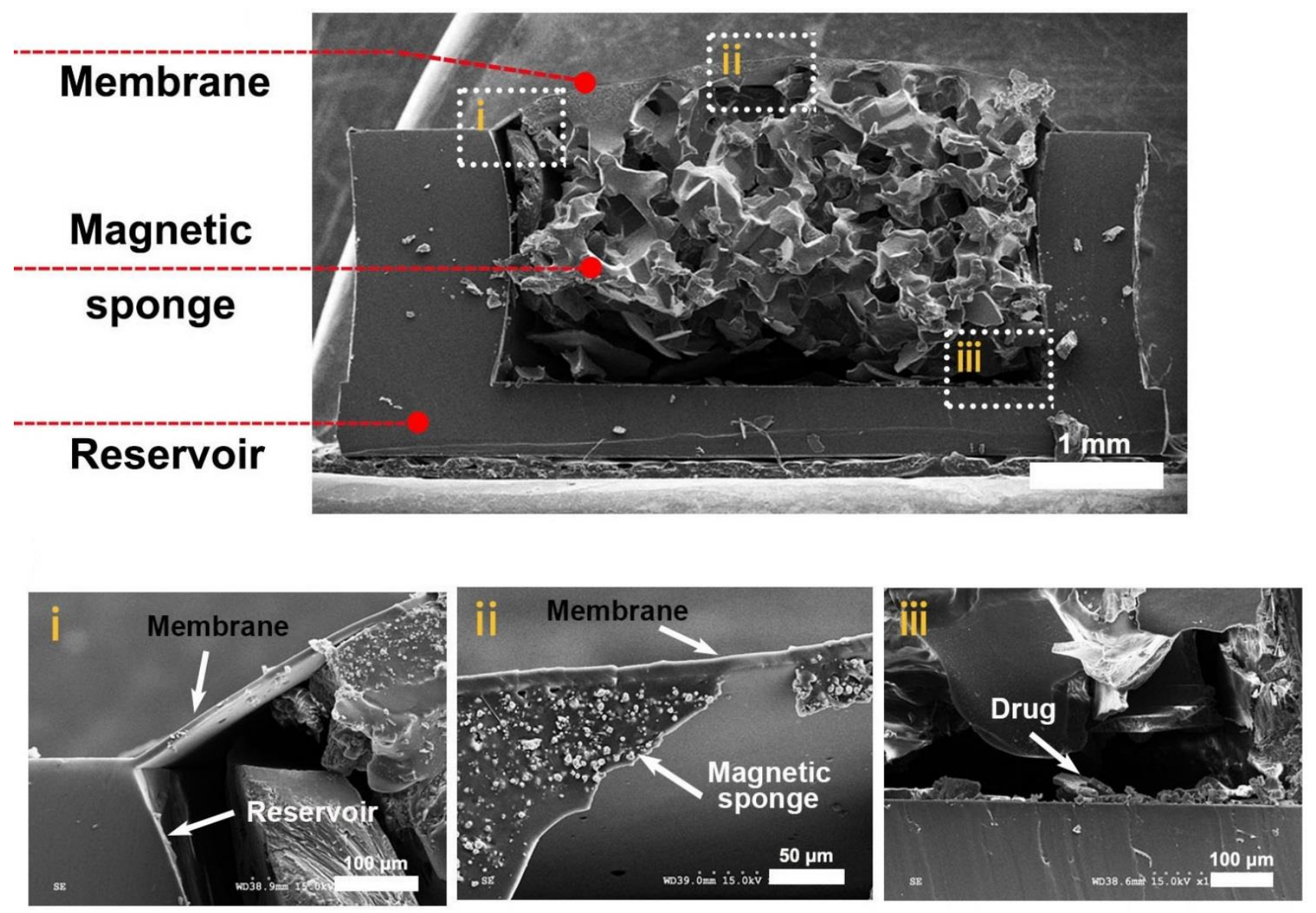

Figure 9- SEM image from cross section of the proposed drug delivery device

\subsubsection{Plasma surface treatment}

In all the aforementioned fabrication processes, air plasma treatment has been performed for 30 seconds, at approximately 700 mtorr pressure using a Harrick plasma chamber (Ithaca, NY, USA). These parameters are determined to provide the strongest bonding and the best surface wettability [46]. 


\subsubsection{Device activation}

As noted earlier, the drug is loaded during the assembling process and prior to placing the magnetic sponge. The reservoir is subsequently sealed by a membrane. In this state, if the device is immersed in a solution or positioned in a biological environment (e.g. tissue), no release occurs. Since the deposited drug is in solid form and has not been dissolved yet, the device is inactive. Device activation takes place when the reservoir is filled with a solvent. Therefore, it is able to inject a solution of the drug.

To activate the device, it is submerged in a designated medium (water or phosphate buffer saline (PBS) including $1 \% \mathrm{w} / \mathrm{v}$ bovine serum albumin (BSA)) and placed in a vacuum chamber for about 10 minutes to vacuum the air exist in the reservoir. By venting the chamber, the medium enters the device through the aperture and dissolves the drug loaded into the reservoir.

\subsection{Device Characterization}

To assess the proposed drug delivery device performance, several tests such as model drug release, real drug release, injected flow study, and ex vivo tissue implantation have been designed and conducted. In this section, each experiment's conditions is demonstrated.

\subsubsection{Methylene blue release}

The first step involves choosing a model drug to test injection behavior. Methylene blue (MB) was selected as the surrogate drug due to its neat spectrometry properties and high solubility. A solution of this substance has been made with the concentration of $10 \mathrm{mg} / \mathrm{ml}$ in water. Each device was then filled with about $20 \mu \mathrm{l}$ of this solution, resulting in $200 \mu \mathrm{g}$ of methylene blue. The medium used to activate the device and perform the release test was water. 
The device is placed at the bottom of a $20 \mathrm{ml}$ vial, and submerged in a $5 \mathrm{ml}$ of distilled water. A $120 \mathrm{mT}$ magnetic field is applied to actuate the device only once for 5 seconds, followed by 10 minutes of a dormant phase. This operation is repeated for 90 minutes to evaluate cumulative $\mathrm{MB}$ injection and test device functionality over several release. Two $1 \mathrm{ml}$ samples were taken out from the solution before and after release to measure the amount of MB released in each actuation. The amount of released MB was quantified by reading the absorbance of the samples at a wavelength of $662 \mathrm{~nm}$ using UV-Vis spectrophotometer (50 BIO, Varian Medical Systems Inc., Palo Alto, CA, USA). The obtained absorbance was converted to the concentration and the amount of MB that existed in the solution. In order to be able to relate the absorbance to the concentration, a standard curve has been obtained by measuring the absorbance of solutions with known concentrations (Appendix B). Figure 10 shows the discharged MB out of the device which travelled about $7 \mathrm{~mm}$ into the medium.

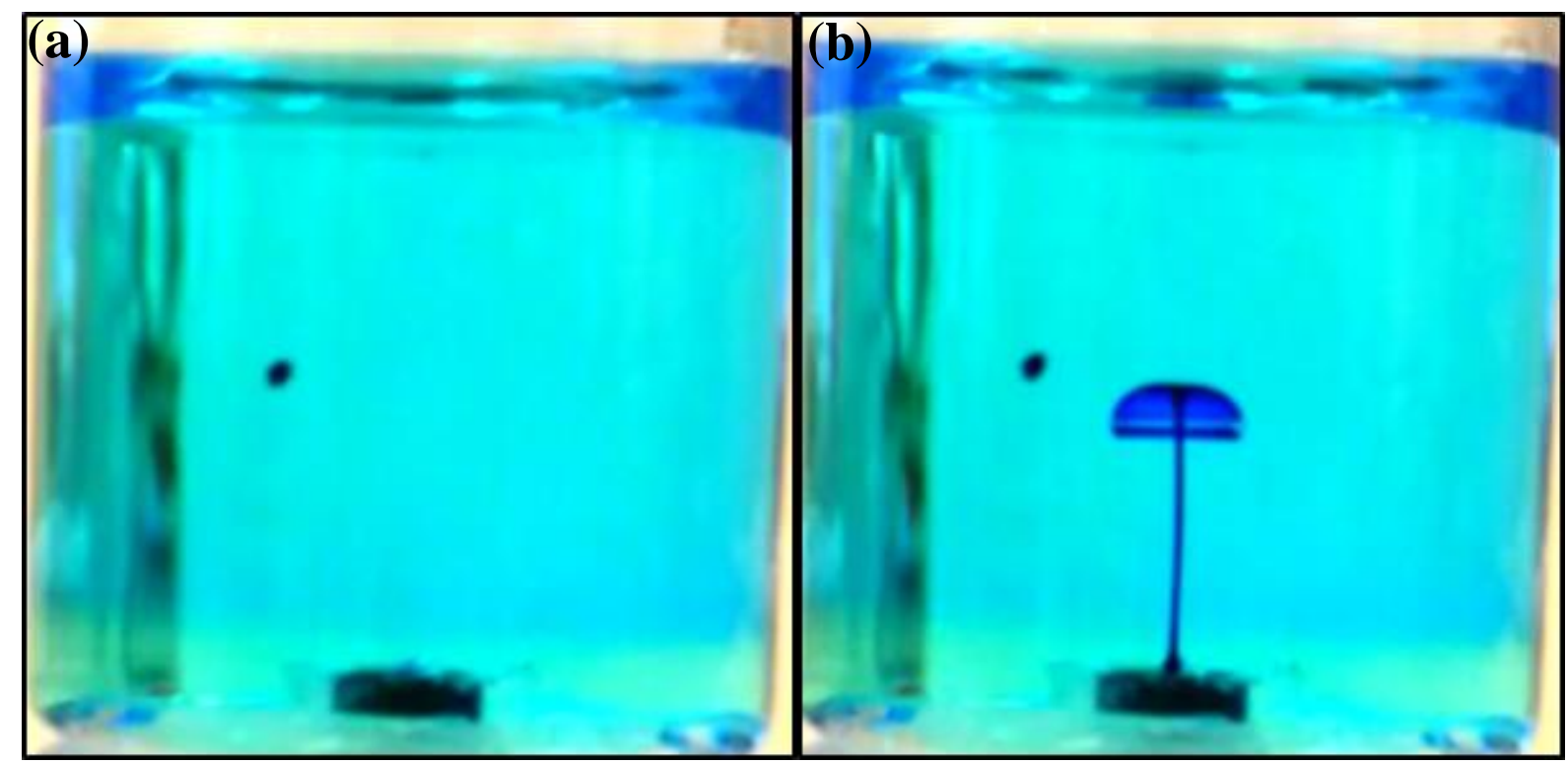

Figure 10- Methylene blue release, a) dormant phase, b) actuation 


\subsubsection{Docetaxel release}

To assess device functionality to release a real drug, docetaxel (DTX) has been selected as a well-established anticancer medication which is used for several cancer disease such as prostate, breast, gastric, head and neck, and non-small cell lung cancer, according to National Cancer Institute (NCI). Docetaxel as a taxane-based drug has anti-proliferative characteristics which hinder and impede cell replication leading to inactivation and death of the cancer tumor cells [47]. Several studies have been conducted to assess the docetaxel treatment efficacy based on different protocols [48]-[50]. Inevitable side effects such as myelosuppression, neutropenia, and nail toxicity have been reported [51]-[53]. Altogether, this drug has provided palatable results in cancer therapy so far.

To prepare the drug solution, 50\% of tritium labelled DTX (50 $\mu \mathrm{Ci} / 200 \mu \mathrm{l})$ in ethanol (Moravek Biochemicals Inc., Brea, CA, USA) was used and mixed with $4 \mathrm{mg}$ of unlabeled DTX in dichloromethane which resulted in $40 \mathrm{mg} / \mathrm{ml}$ of DTX solution. Each device was then loaded with $400 \mu \mathrm{g}$ DTX, and $50 \mu \mathrm{g}$ MB which was used as a visual aid to detect injection. Since DTX solubility has been reported low in water (around $5 \mu \mathrm{g} / \mathrm{ml}$ ) [54], phosphate buffer saline (PBS, $\mathrm{pH}$ 7.4) including $1 \%$ bovine serum albumin (BSA) (subsequently referred as PBS/BSA solution) was chosen as the medium for docetaxel release test as DTX has shown better solubility in this solution [32]. The device is situated at the bottom of a $20 \mathrm{ml}$ vial in a $4 \mathrm{ml}$ PBS/BSA solution and actuated for 5 times, each of which consists of 7 seconds of on magnetic field followed by 5 seconds of relaxation when the device was not actuated. This actuation interval, which takes about one minute, was repeated for 2 hours with 20 minutes layoff. DTX release in three consecutive days has also been studied and the results are provided in the next 
section. Low solubility of DTX might make the detection difficult. Thus, longer and more actuation protocol was opted for increasing the amount of released DTX.

Two samples of $500 \mu \mathrm{L}$ were extracted before each actuation interval to measure the background DTX concentration of solution. After the actuation interval, three samples of $500 \mu \mathrm{L}$ were pipetted into the scintillation vials. Each sample took one scintillation vial which was earlier filled with about $5 \mathrm{ml}$ of Cytoscint liquid scintillation fluid (Fisher Scientific, Fair Lawn, NJ, USA). The scintillation vials were then stirred using a vortex mixer and the disintegrations per minute (DPM) was read by a liquid scintillation counter (Tri-Carb, PerkinElmer, Waltham, MA, USA). The standard curve had previously been provided from specified specimens with predetermined concentrations, enabling us to convert and relate the raw DPM data to the amount of DTX exists in the solution in terms of ng. Three scintillation vials with $5 \mathrm{ml}$ of scintillation fluid, which were free of DTX, were used to measure the background radiation. The associated number was later subtracted from the data received from DTX release, in order to merely consider and compute the DTX effect only.

\subsubsection{Injected flow study}

As depicted in Figure 10, this device releases the drug like a jet flow and considerably far from the device. To study the behavior of this jet flow, device injection has been captured by a highspeed camera. This test was carried out in three different magnetic field strengths to investigate the influence of magnetic fields on the injection. Position of the tip of injected MB was computed by image processing of the extracted frames using Matlab. Therefore, injection depth versus time has been achieved. As a consequence, the tip velocity has also been estimated using 
a numerical differentiation method. However, obtaining the exact velocity demands more accurate techniques.

\subsubsection{Ex vivo tissue implantation}

To assess the feasibility of device implantation and to test drug release inside tissues, DTX loaded devices were placed inside the porcine bladder tissues. Porcine bladder tissues were cut into the size fitting the device. The tissue interior was washed and filled with PBS/BSA solution and the device was implanted. The device including the tissue was exposed to a $280 \mathrm{mT}$ magnetic field and actuated twice. Each actuation was associated with 5 seconds of magnetic field exposure followed by 7 seconds resting.

After actuation, the targeted site of each tissue stained by discharged MB was cut and immersed in an aqueous-based tissue solubilizer (Solvable, PerkinElmer, Inc., Waltham, MA, USA) in a scintillation vial. The solution was left for about one day to digest the tissue sample. Subsequently, $3 \mathrm{ml}$ of scintillation fluid was added to the mixture and stirred using a vortex mixer. The radioactivity of each sample was then evaluated similar to the method used for DTX release experiment. 


\section{Chapter 3: Results and Discussions}

In the previous chapter, device fabrication and subsequent experiments carried out to qualify device performance have been demonstrated. This chapter is dedicated to the data obtained from the described tests and discusses the results. Firstly, methylene blue release test is shown. Docetaxel release, injected flow behaviour, and ex vivo tests are later presented, respectively.

\subsection{Methylene Blue Release}

Methylene blue is a highly soluble substance in water. Its solubility has been reported around 40 $\mathrm{mg} / \mathrm{ml}$ in water by the manufacturer. Hence, it is expected that the amount of MB in the reservoir decreases as the number of actuation increases or due to consecutive actuation. However, ease of use and its excellent spectrometry characteristics make it a favorable candidate as a surrogate drug. Besides, unlike the docetaxel, it does not have any radioactive properties, making its handling much simpler.

As depicted in Figure 11, this device released $2.28 \pm 0.23 \mu \mathrm{g}$ MB per actuation $(\mathrm{n}=36)$ in a 120 $\mathrm{mT}$ magnetic field strength which is equivalent to the distance of $6 \mathrm{~mm}$ between device and the magnet. As mentioned in previous section, each device was filled with $200 \mu \mathrm{g}$ MB. Considering the released amount, this implies that the device will be depleted after about 87 actuations in this magnetic field. 


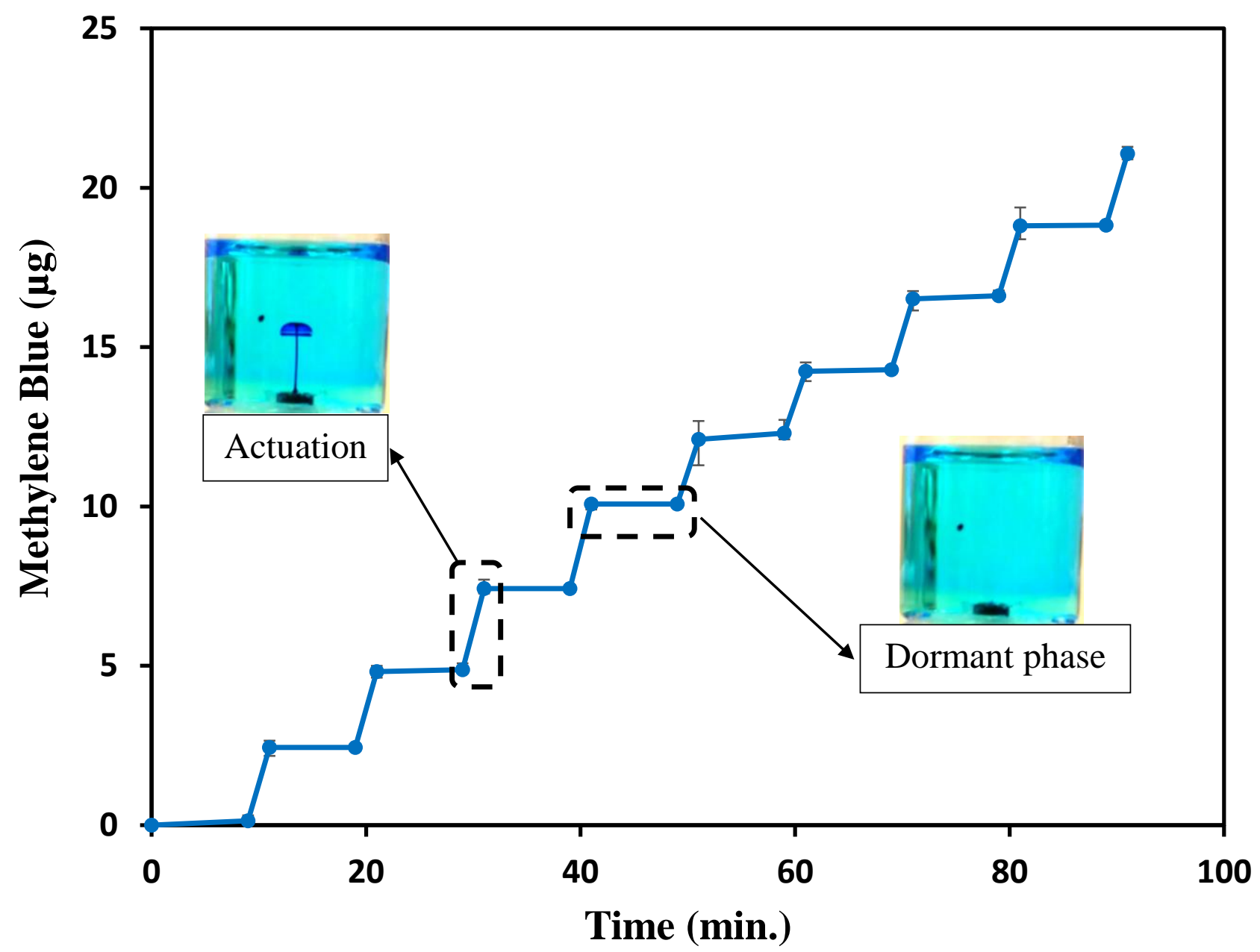

Figure 11- Cumulative methylene blue release over time

\section{Influence of the magnetic field:}

Another factor affecting the amount of released MB is the strength of magnetic field. Stronger magnetic fields exert more force on the magnetic sponge. Although the magnitude of force is proportional to the magnetic properties of the sponge (i.e. the concentration of the CI magnetic microparticles), the ultimate sponge shrinkage is dictated by its elasticity modulus as illustrated in section 2.3.2. However, for one particular magnetic sponge, stronger magnetic field leads to more shrinkage which results in more drug injection. 
In order to evaluate the effect of applied magnetic field, the device was exposed to different magnetic field strengths and the consequent release was quantified. However, other conditions such as the medium (water) and etc. remained unaltered as described for MB release in section 2.5.1.

The results shown in Figure 12 indicates that more MB released as the magnetic field strength increases. Thus, the magnetic field strength can potentially be used as a critical parameter to adjust the amount of introduced drug according to the treatment protocol.

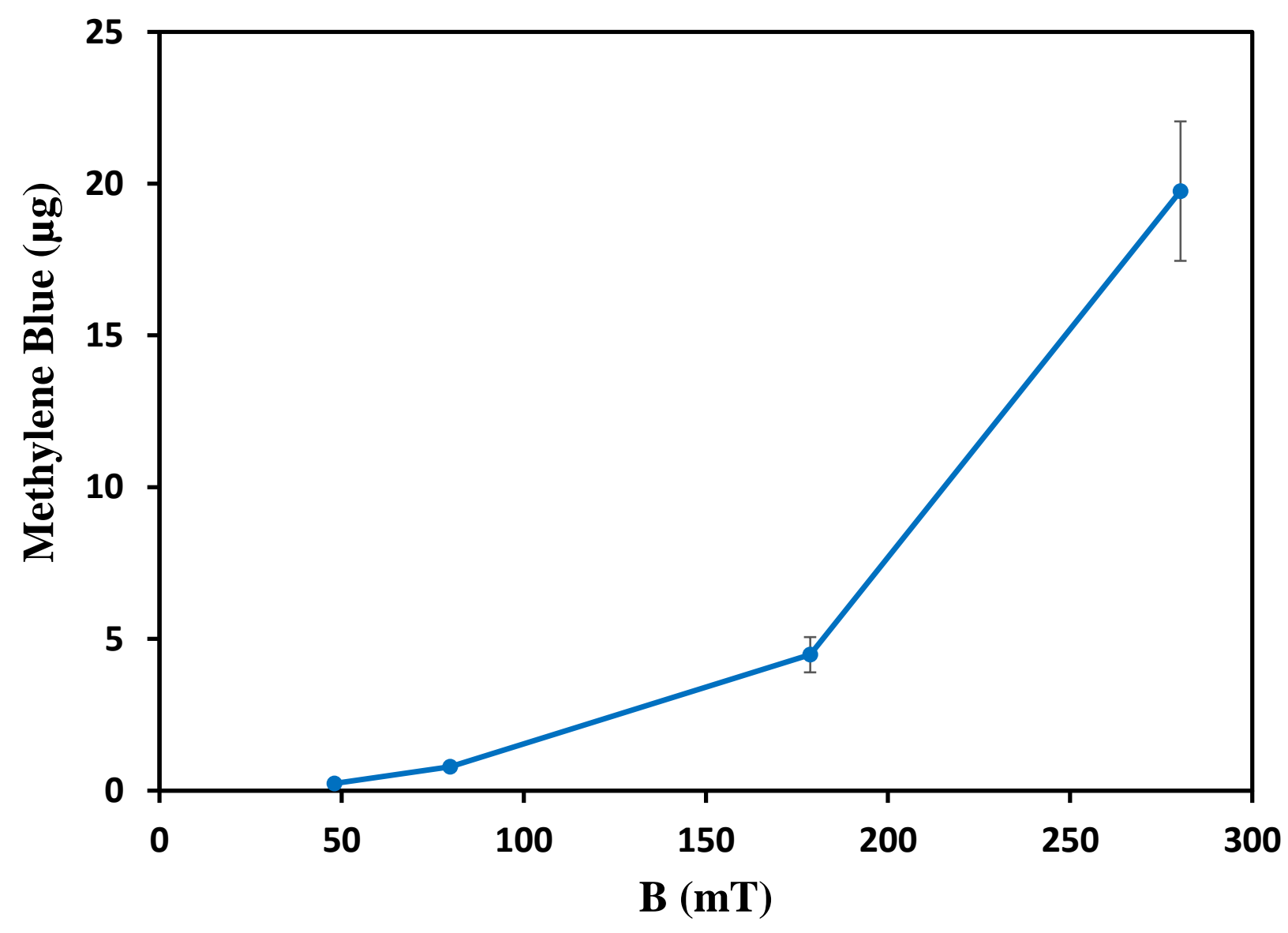

Figure 12- MB release in various magnetic field strength 


\subsection{Controlled Docetaxel Release}

Since the docetaxel is colorless, MB was merely used to visually confirm the injection ensuring drug release. As mentioned in section 2.5.2, the radioactivity of released DTX is measured and later converted to the amount of DTX. The radioactivity's parameter used as the measurement variable is called disintegration per minutes which basically is the number of radioactive atoms decay in one minute. For instance, the background radiation was measured to be around $20 \mathrm{dpm}$. The tritium, which was used in our case as the radioactive label for DTX, has a low radioactivity. Thus, in order to prevent any interference between DTX detection and the background radiation, one way is to increase the number of tritium labeled DTX. Hence, the device was actuated more to raise the amount of DTX in the solution and make the samples more radioactive. Due to low solubility of DTX, the dormant phase was also selected longer (20 minutes) to ensure that the DTX was dissolved thoroughly in the reservoir for the next actuation.

\subsubsection{Experimental results}

Cumulative DTX release is presented in Figure 13, which exhibits $40.34 \pm 4.23$ ng DTX in each actuation interval. To evaluate device release consistency, this trend was repeated several times. Low amount of DTX stems from its low solubility.

The device was also actuated for about 3 hours in three consecutive days. After each actuation period, the device sat in PBS/BSA solution for the rest of day until next actuation (i.e. next day). The results is plotted in Figure 14, which shows roughly $350 \mathrm{ng}$ DTX release in each day. As explained before, each device contained about $400 \mu \mathrm{g}$ DTX including $50 \mu \mathrm{g} \mathrm{MB}$. Therefore, this device released only about $0.01 \%$ and $0.088 \%$ of the drug supply in each actuation interval and each day, respectively. In other words, the device will be depleted in 10000 actuation interval. 
Nonetheless, it is believed that the designated reservoir is capable of storing even more drug up to $1 \mathrm{mg}$. Thus, there is no concern regarding depletion of the drug in short term. However, the suitable amount of drug is determined according to the treatment protocol and the course of therapy.

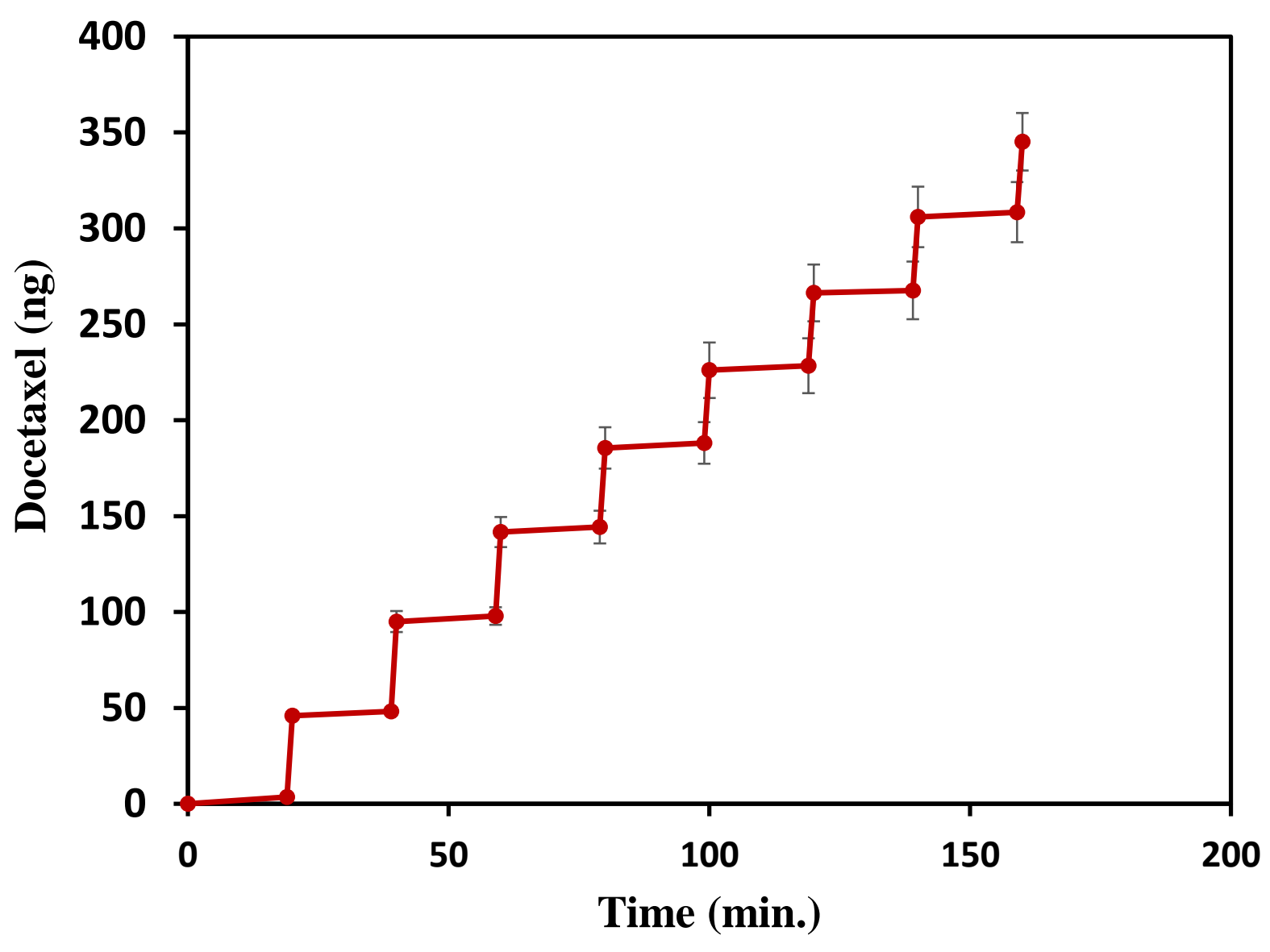

Figure 13- Cumulative docetaxel release in a $180 \mathrm{mT}$ magnetic field strentgh 


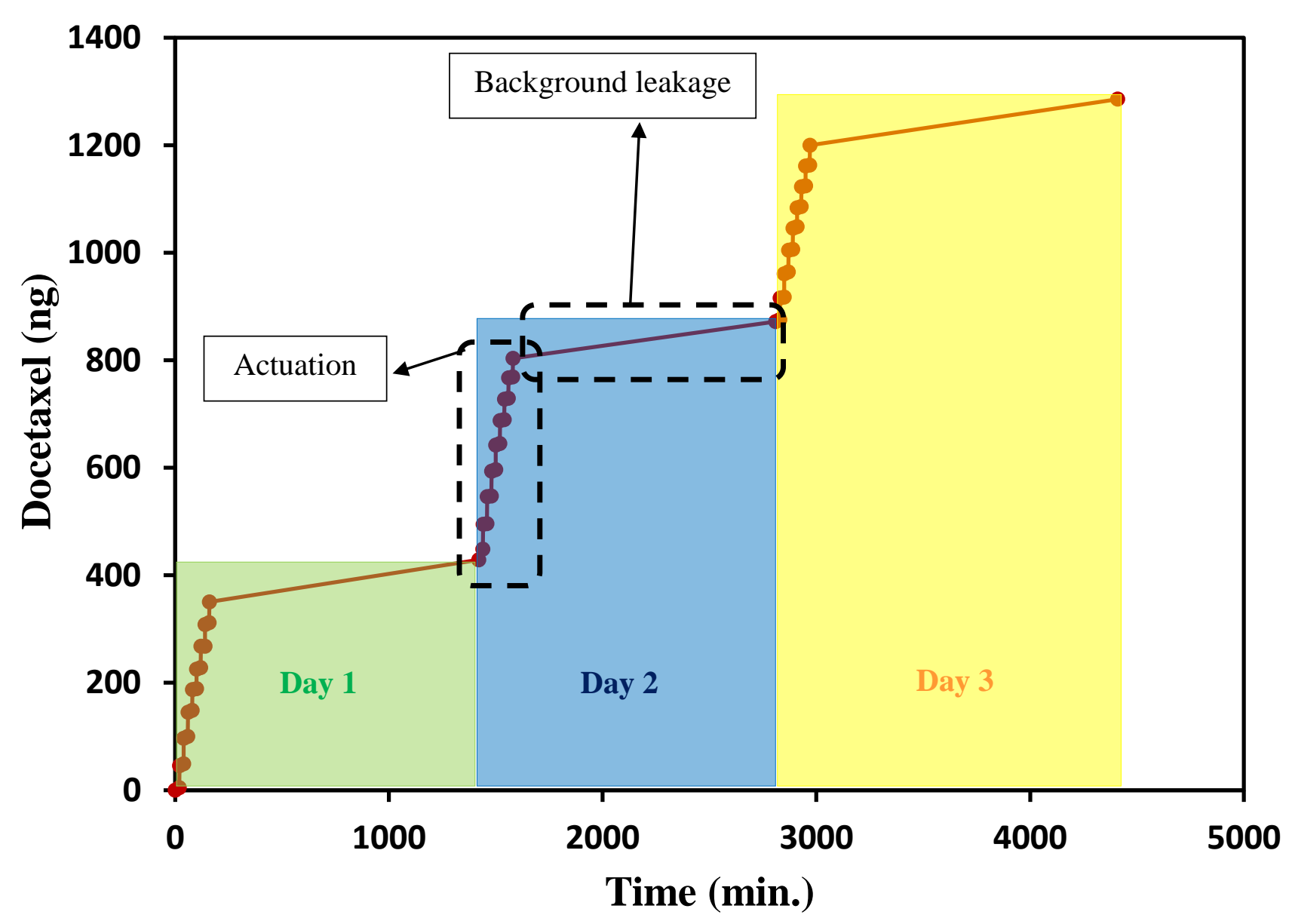

Figure 14- Three consecutive day docetaxel release

\subsubsection{Background leakage:}

In the device fabrication process, an aperture is created by laser ablation which means that there is a permanent opening in device. Although the main objective of this aperture is to redirect the drug out of the reservoir once device is actuated, the drug can leave the device while no actuation occurs. This phenomenon is due to the much higher drug dosage inside the device which leads to drug diffusion through the surrounding ambient with much lower concentration. This is, one of 
the features of the reservoir-based drug delivery devices which incorporate apertures without valves. In this device, because of the interconnected pores of sponge, drug solution is somehow entrapped within the sponge structure impeding the leakage. Due to the high concentration gradient, this diffusion could not be ceased unless a valve is designated for the aperture.

In order to find out the rate of background leakage, the device was left in PBS/BSA solution for more than 20 hours. However, to compute the leakage rate, the initial and final concentration of the solution were acquired by taking out two and three $500 \mu \mathrm{l}$ samples at beginning and end of test, respectively. This device has background leakage of $0.060 \pm 0.004 \mathrm{ng} / \mathrm{min}(\mathrm{n}=4)$ which can also be seen in Figure 14 as the line connecting two consecutive actuation period. In localized drug delivery, this leakage occurs in the target zone (e.g. tumor).

\subsubsection{In vitro cell study}

A similar drug was used in a different drug delivery device and the corresponding DTX activity on the PC3 prostate cancer cells has been achieved [30]. As revealed in Figure 15, cell death commenced at around $10 \mathrm{ng} / \mathrm{ml}$ concentration, which drastically decreased cell proliferation by $60 \%$. However, the cell viability trend declined to around $35 \%$ at a higher concentration of 52 $\mathrm{ng} / \mathrm{ml}$. Thus, nano-concentration of this drug is adequate for the treatment. Furthermore, since the amount of released DTX in previous section was computed for a $3 \mathrm{ml}$ medium, the concentration of released DTX is about $13.45 \mathrm{ng} / \mathrm{ml}$, which is within the active range of the DTX (more than $10 \mathrm{ng} / \mathrm{ml}$ ). The amount of introduced drug can be adjusted to attain the favorable dosage. 


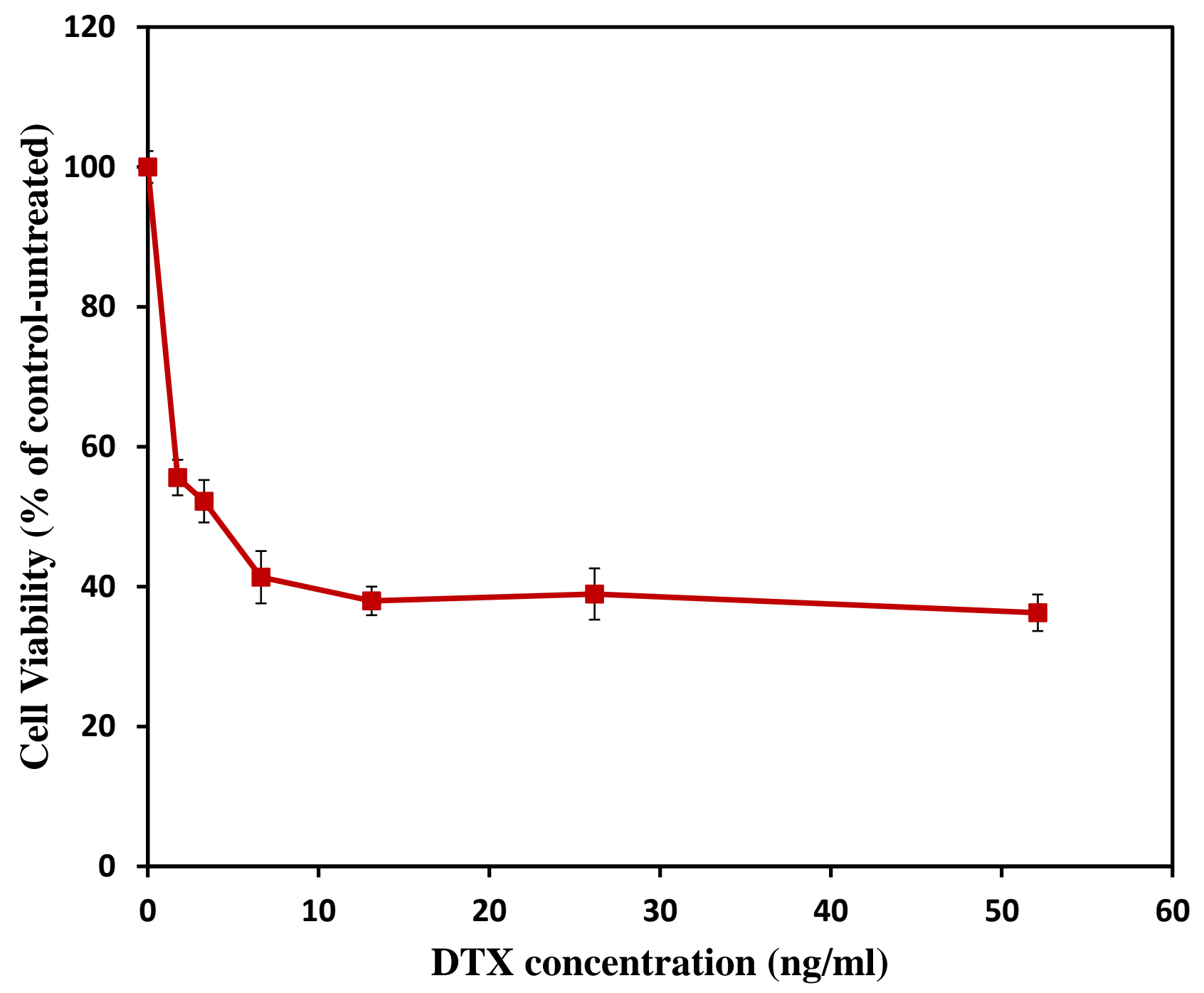

Figure 15- Inhibition of PC3 cell proliferation using docetaxel [30] 


\subsection{Injected Flow Study}

In this device, the drug administration can be controlled by tuning the applied magnetic field, which controls the drug dosage. Moreover, the magnetic field strength affects the depth of injection (Figure 10) which took place at a $120 \mathrm{mT}$ magnetic field. Since the injected drug travels a relatively long path in a short time, it may be considered as a jet flow.

The injection depth versus time has been plotted in Figure 16 for three different magnetic field strengths. The maximum travelled distance is about $4.5 \mathrm{~mm}, 7.5 \mathrm{~mm}$, and $10 \mathrm{~mm}$ in a $97.4 \mathrm{mT}$, $117.2 \mathrm{mT}$, and $144.7 \mathrm{mT}$ magnetic field strength respectively. As illustrated in the results, the magnetic field strength is able to directly affect both the introduced drug dosage and the depth of injection. This latter unique characteristic signifies that the drug is able to reach the target. Moreover, the drug is injected far enough from the device that the majority of it will not be pumped back into the reservoir once the magnetic field is removed. Thus, most of the released drug will remain in the target zone and diffuse by passage of the time. The diffusion time is a chemical property of the prescribed medicine.

The velocity of this jet flow could be estimated by numerical analysis of the data obtained for the tip position versus time. In this aspect, numerical differentiation was taken from the data points using five point midpoint method. In this method, a polynomial of degree 4 is fitted to the first 5 points. Then, the amount of midpoint derivative is calculated by differentiating from the curve equation. This trend continues by shifting the group point, one point forward and applying the same process (curve fitting) to compute the next midpoint derivative (which is the next adjacent point of previous point). In this method, the second data point derivative is evaluated by differentiating the curve fitted to the first five points. However, the associated error is theoretically more than the midpoint's. Although this method is also practical with different 
number of points (e.g. $3,5,7, \ldots)$, increasing the number points does not necessarily lead to better results. In fact, increasing the number of selected points will increase the conditions of curve fitting and might cause inappropriate fitting which could consequently result in more error in calculated velocity. Furthermore, increasing the number of fitting points will prolong the computation time. Thus, there is a tradeoff between the number of adopted points and error of estimation. In order to choose the suitable number of points, the maximum velocity obtained from three different cases of 3,5 , and 7 points, have been compared for a $117.2 \mathrm{mT}$ magnetic field. From three to five points, the maximum velocity changed about $1.75 \%$ and from 5 to 7 points this change was roughly $0.34 \%$. Therefore, due to the very low change in 7 points, 5 point method has been selected.

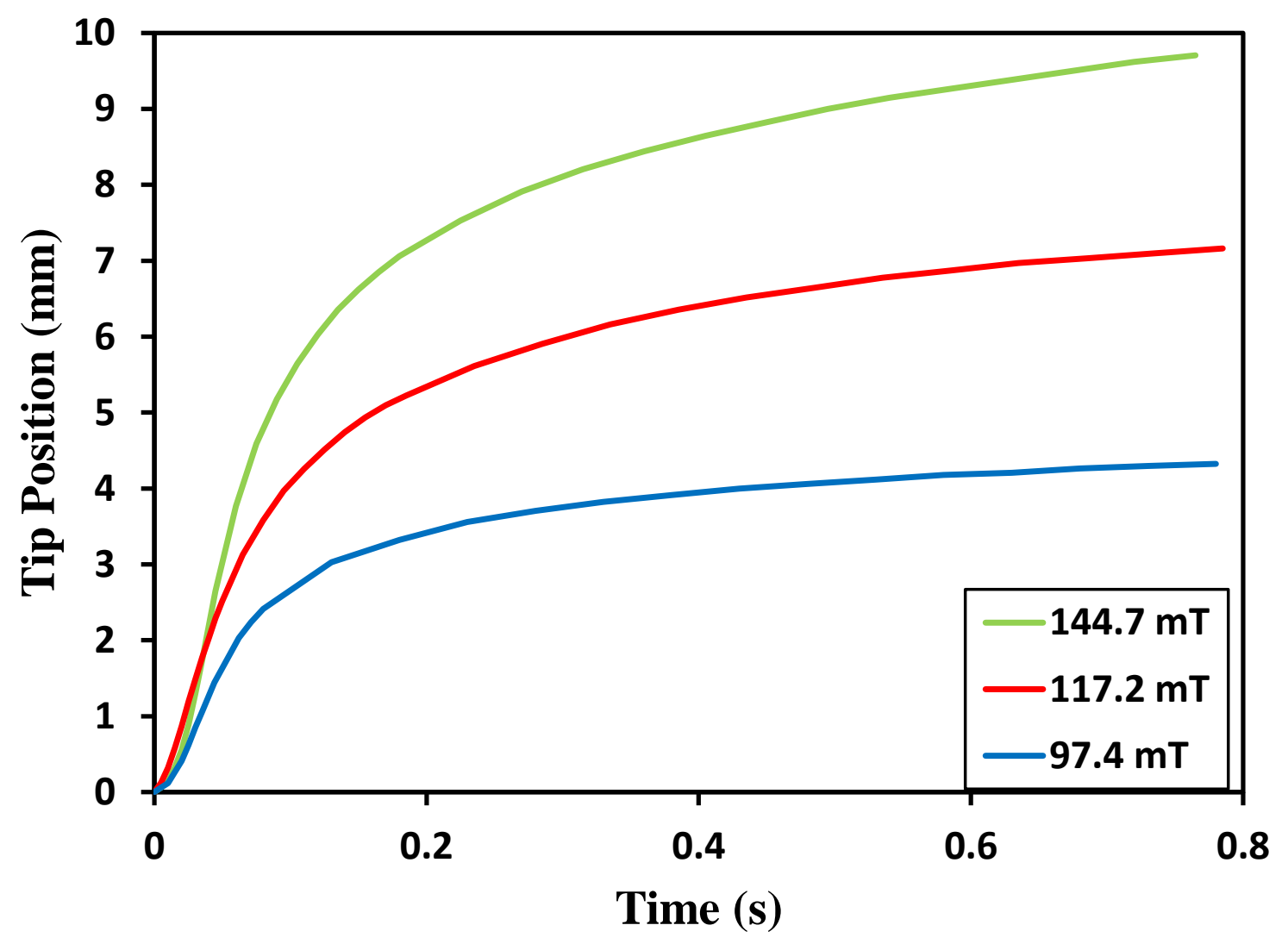

Figure 16- Position of tip of injected flow versus time 
High injection velocity is able to push the protein away and prevent aperture clogging from biofilm formation. If it is necessary to obtain precise velocities, further investigation employing more accurate methods such as particle image velocimetry (PIV) is required. As a result, the jet flows reached the maximum velocity of approximately $45 \mathrm{~mm} / \mathrm{s}, 62 \mathrm{~mm} / \mathrm{s}$, and $92 \mathrm{~mm} / \mathrm{s}$ in the aforementioned magnetic field strengths respectively.

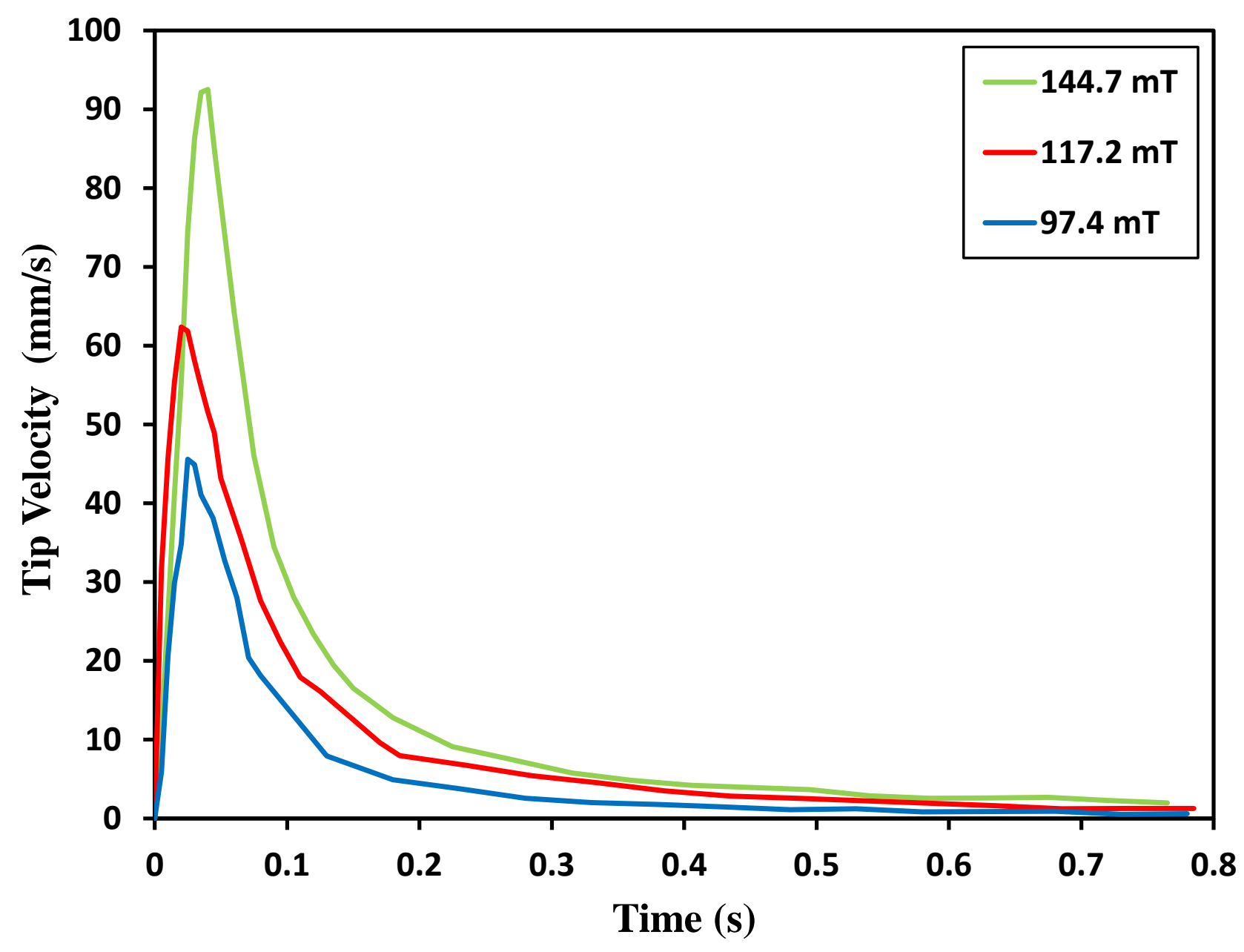

Figure 17- Estimated tip velocity of the jet flow versus time 
The generated shear stress has also been estimated when the jet flow reaches its high velocity. For instance, in a $117.2 \mathrm{mT}$ magnetic field strength, injected flow reached about $62 \mathrm{~mm} / \mathrm{s}$ in 20 ms and travelled about $0.9 \mathrm{~mm}$. As illustrated in Figure 18, following assumptions have been applied to compute the shear stress:

1. The injected MB is assumed to be symmetric with the maximum width of $w(\Delta y=w / 2)$,

2. The maximum velocity is located at the center of the released MB.

3. The medium is assumed to be stationary. As a result, the velocity at the side boundary of jet flow and medium is considered zero

The shear stress is calculated for different cases according to the following equation:

$$
\tau=\mu \frac{\Delta V}{\Delta y}=\mu \frac{V_{\max }-v}{w / 2}=\mu \frac{2 V_{\max }}{w}
$$

where $\tau$ is the dynamic viscosity of water which is equal to 0.001 Pa.s at room temperature. The maximum velocity, width, and the consequent shear stress have been provided in the Table 2 for various cases.

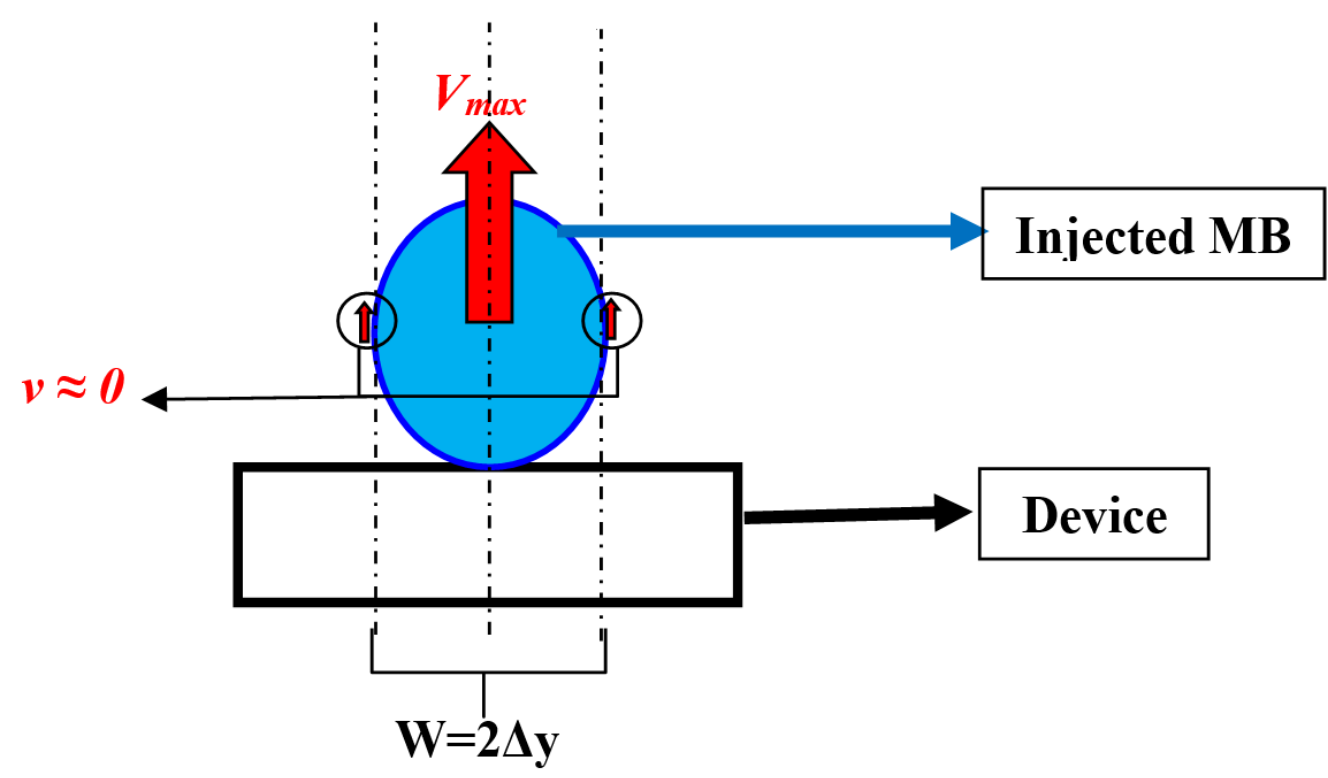

Figure 18- Schematic view of the injected MB and involved parameters 
Table 2- Estimated shear stress for different magnetic field strengths of $97.4 \mathrm{mT}, 117.2 \mathrm{mT}$, and 114.7mT

\begin{tabular}{|c|c|c|c|}
\hline $\begin{array}{c}\text { Magnetic Field } \\
(\mathrm{mT})\end{array}$ & $\begin{array}{c}\text { Maximum Velocity } \\
(\mathrm{mm} / \mathrm{s})\end{array}$ & $\begin{array}{c}\text { Maximum width } \\
(\mathrm{mm})\end{array}$ & $\begin{array}{c}\text { Estimated shear stress } \\
\left(\mathrm{dyne} / \mathrm{cm}^{2}\right)\end{array}$ \\
\hline 97.4 & 45.6 & 0.74 & 1.23 \\
\hline 117.2 & 62.4 & 0.81 & 1.54 \\
\hline 144.7 & 92.5 & 0.88 & 2.1 \\
\hline
\end{tabular}

As an analogy, the shear stress required to desorb the BSA molecules from a Ti coated surface was obtained about 0.037 and $0.35 \mathrm{dyne} / \mathrm{cm}^{2}$ [55], which is in the same order of magnitude of our estimated shear stress. However, device configuration might require modification in order to fulfill the requirement of implant which might consequently affect the obtained data. Although a precise method is required to accurately acquire the velocity profile and the shear stress, this feasibility study shows that this device is potentially able to remove the possible accumulated protein on the aperture. 


\subsection{Ex Vivo Tissue Implantation}

Device implantability was assessed by investigating release behavior while the device was located in porcine bladder tissue. In order to protect the membrane form direct contact of tissue, a $1 \mathrm{~mm}$ thick housing was made by PDMS and attached to the top of the reservoir's wall. The perfect shape and dimension of housing could be determined later once device is ready for an in vivo study.

This device injected about $13.61 \pm 2.27 \mathrm{ng}$ docetaxel to the targeted tissue in a $280 \mathrm{mT}$ magnetic field with twice actuation. On the other perspective, sample tissues' mass were also logged (varied between $0.33-0.58 \mathrm{~g}$ ), which with the density of around $1 \mathrm{~g} / \mathrm{ml}$, results in docetaxel concentration of $33.21 \pm 5.49 \mathrm{ng} / \mathrm{ml}$. However, according to the PC3 cell study shown in Figure 15 , docetaxel activity begins at around $10 \mathrm{ng} / \mathrm{ml}$. Furthermore, the device was also subjected to 4 times actuations in the same magnetic field increasing the released docetaxel to $37.39 \pm 8.75 \mathrm{ng}$. Hence, this device has demonstrated effective drug release during implantation that provides adjustable injection, which is a function of the magnetic field strength and the number of actuations. Results including images of the experiment are presented in Figure 19. 

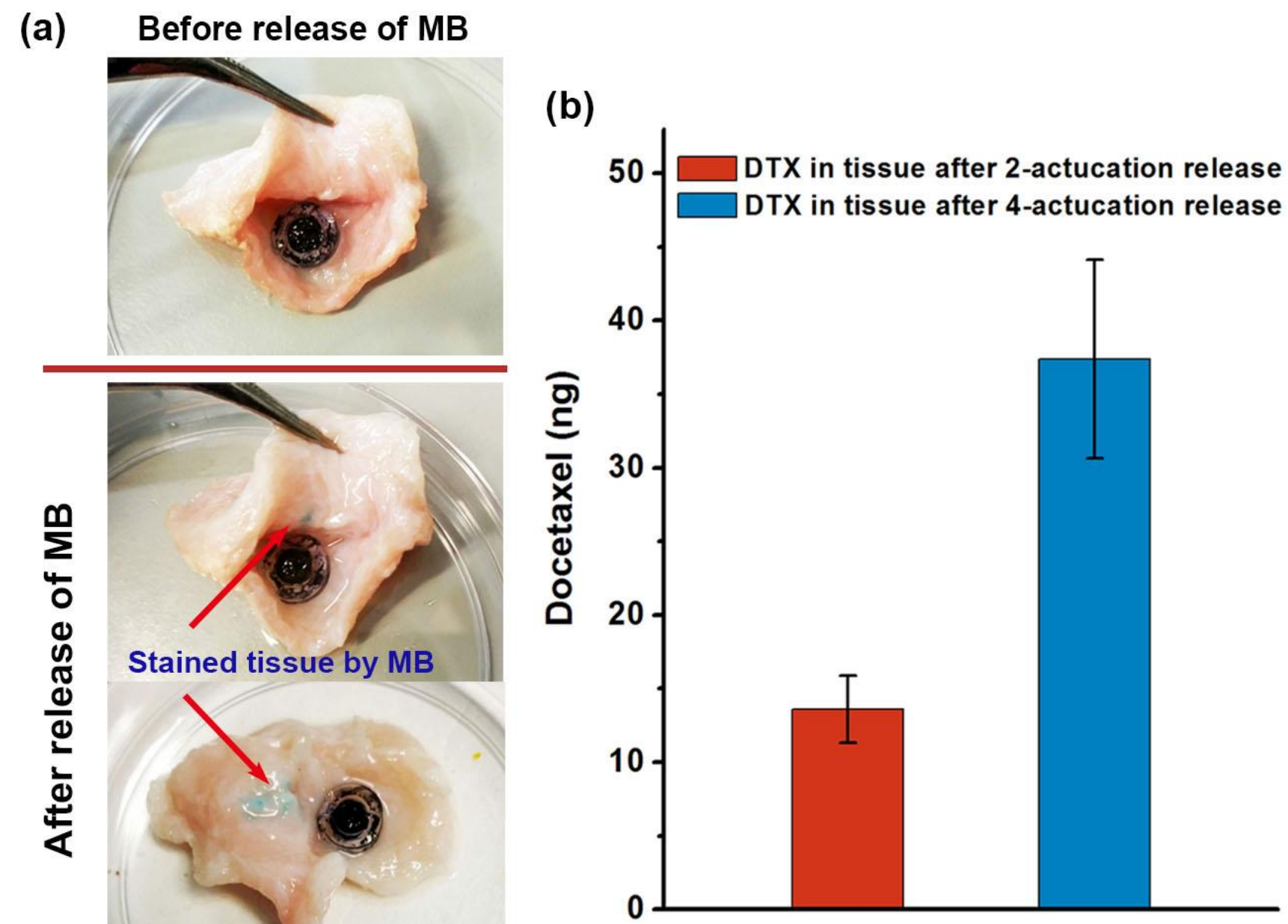

Figure 19- a) Implanted device inside a porcine bladder tissue, before and after actuation are shown, the blue stain is caused by the methylene blue loaded in the reservoir in addition to docetaxel, b) Measured docetaxel in the targeted site of the tissues after 2 and 4 times actuation 


\section{Chapter 4: Conclusions and Future Work}

Treatment efficiency depends on the type of therapeutic agent and its dosage effectively delivered to the disease site such as tumor. Several successful methods and creative ideas concerned with drug delivery have been reviewed and major persisting obstacles have been discussed in the Introduction Chapter. This thesis focused on introducing a novel approach towards targeted drug delivery by taking into account the existing issues and is based on a biocompatible magnetic sponge.

The drug-containing magnetic sponge is constructed from polydimethylsiloxane and is deformed when an external magnetic field is applied which leads to discharge its content. This sponge is able to retain its initial condition once the magnetic field is removed, and applying on/off magnetic fields result in effective pumping in/out solution. Furthermore, sponge shrinkage can be controlled by tuning the magnetic field strength. The magnetic sponge may absorb the therapeutic compound by submerging in a drug solution. Nonetheless, a device has been fabricated composed of a reservoir, magnetic sponge, and sealing membrane. This device obviates the need for power, and is able to release the drug on-demand at the prescribed dosage by applying a specified magnetic field.

Various dosages of DTX in the range of $30-75 \mathrm{mg} / \mathrm{m}^{2}$ body-surface area have been systemically administrated [48]-[50], [56], [57] for tumor treatment. Body-surface area is a unit defined to allot suitable dose of the drug to each individual with respect to their own weight and height. In this regard, different formulae have been proposed so far to calculate the value [58]. As a rough estimation, if the prescribed dose and the body surface area of one typical patient are considered $30 \mathrm{mg} / \mathrm{m}^{2}$ and $2 \mathrm{~m}^{2}$ respectively, the amount of DTX introduced to body in direct administration 
would be $60 \mathrm{mg}$. As mentioned earlier, this amount of DTX will spread through the whole body and affect other healthy organs. Localized delivery of DTX will require less dosage [59]. According to the experimental data obtained in DTX release test and cell study, only 2 ng DTX is sufficient to inhibit cell proliferation to roughly $40 \%$ ( $200 \mu \mathrm{l}$ of each concentration was used [30]).

Moreover, on the release test with the defined actuation criteria, this device released around 40 ng of DTX which corresponds to approximately $70 \%$ decrease in cell viability. By comparing these two numbers, localized drug delivery is theoretically able to substantially diminish the amount of DTX introduced to body. By focusing on the defected zone or tumor, nano-dosage of the drug should potentially be adequate for the treatment. Besides, other organs will be immune to the side effects of the medication as they are not exposed. Further rigorous investigations are required to firmly establish this hypothesis.

This thesis began with an introductory chapter which covered the literatures concerned with drug delivery methods. This was followed by the second chapter focusing on the fabrication process of the magnetic sponge and the subsequent proposed drug delivery device. This chapter also introduced the experiments carried out to test device performance. The third chapter reported the results obtained from the described experiments and analyzed data to assess device function as a drug delivery system. The final chapter summarizes the work, concludes the results, and presents future views on the development of the proposed drug delivery device.

\subsection{Summary}

Chapter 1 presents the efforts that have been made in the field of drug delivery, particularly the localized delivery of the therapeutic agent. 
Chapter 2 has introduced the new method of drug delivery which is based on a magnetic sponge. First of all, the fabrication process of magnetic sponge is reviewed in details. Magnetic sponge fabrication starts with mixing PDMS with carbonyl iron (CI) to achieve magnetic PDMS which, subsequently, becomes porous by pouring it onto the sugar lump. However, different solute agent (e.g. salt) may be used to create different size of pore. The magnetic sponge shrinkage has been determined as the main criteria to evaluate best CI/PDMS w/w ratio. The shrinkage has found to be dependent on two competing factors: magnetic properties and the elasticity modulus of the sponge. However, both of these factors rely on the CI concentration. Although increasing the $\mathrm{CI} / \mathrm{PDMS}$ ratio enhances the magnetic properties, this also results in more elasticity modulus which resists and opposes the exerted magnetic force. Hence, the best CI/PDMS ratio was obtained to be $100 \%$ among two other ratios of $50 \%$ and $150 \%$, which exhibited more displacement in various magnetic fields according to the described deformation test. Later, device fabrication process including the reservoir, and membrane was presented. Reservoir was made with PDMS using a mold which was designed in Solidworks and created by a 3D printer. Total dimension of the reservoir is the thickness of less than $2 \mathrm{~mm}$, inner diameter of $4 \mathrm{~mm}$, and depth of about $1.5 \mathrm{~mm}$. The reservoir has enough space to be loaded with the drug and to encompass the magnetic sponge. The membrane (thickness of about $10 \mu \mathrm{m}$ ) was made by PDMS, on top of a glass slide coated with a sacrificial layer (Polyacrylic acid). The proposed drug delivery device is assembled according to the following steps:

1. Drug is deposited into the reservoir, and, subsequently, the magnetic sponge $(1.5 \mathrm{~mm}$ thick and $4 \mathrm{~mm}$ diameter) is placed inside the reservoir.

2. Plasma surface treatment is applied to the reservoir including sponge and the membrane, in order to covalently bond these components to each other. 
3. Device is set free by dissolving the sacrificial layer with water.

4. An aperture of size of $90 \times 90 \mu \mathrm{m}^{2}$ as a drug exit, was created by laser ablation Subsequently, the conducted experiments have been described after covering device fabrication. The methylene blue release test has been performed as a surrogate drug release test which was followed with docetaxel release test. Docetaxel has been chosen as a well-known anticancer medicine in chemotherapy where its features have briefly been highlighted earlier. Injected flow behaviour and numerical analysis were also studied and carried out to investigate magnetic field strength influence and estimate the injection velocity, respectively. Eventually, the device was implanted inside a porcine bladder tissue to inspect drug release in an ex vivo condition.

Chapter 3 reported and discussed the results gained from the previously defined experiments. This device released about $2.3 \mu \mathrm{g}$ methylene blue in a $120 \mathrm{mT}$ magnetic field with only one actuation. However, this release could increase to roughly $20 \mu \mathrm{g}$ by rising the magnetic field strength to $\sim 280 \mathrm{mT}$. Regarding DTX release, the device discharged about 40 ng DTX in each actuation interval in a $145 \mathrm{mT}$ magnetic field. Three consecutive day DTX releases shows consistent daily DTX discharge of about $350 \mathrm{ng}$. Since the amount of released drug is correlated with the strength of the magnetic field, an equation can be defined to estimate the released dosage of a drug according to the applied magnetic field strength. However, this also depends on the type of the drug and its chemical properties such as solubility and diffusion rate. In order to have a successful drug pumping, the maximum pulse rate of actuation is recommended as $1 / 5$ $\mathrm{Hz}$, however, the solubility and diffusion rate of the drug are the two limiting factors dictating the amount of released drug in each actuation.

The numerical analysis of injected flow indicates that the penetration depth and jet flow velocity are proportional to the strength of applied magnetic field. The maximum estimated velocity and 
travelled distance were achieved about $92 \mathrm{~mm} / \mathrm{s}$ and $10 \mathrm{~mm}$, respectively, in a $\sim 145 \mathrm{mT}$ magnetic field. However, the travelled distance reported by Pirmoradi et al. was $220 \mu \mathrm{m}$ in a 170 mT magnetic field strength [30] which has improved by Zachkani et al. to roughly $1.3 \mathrm{~mm}$ under a magnetic field of $136 \mathrm{mT}$ [60]. As mentioned before, higher velocity and longer depth of penetration can potentially be effective in removing the biological material (e.g. protein) agglomerated around the device and delivering the drug to the target point. However, these preliminary results could be a first step in accurately characterizing discharged flow behaviour using more precise methods (such as particle image velocimetry).

The device was successfully implanted inside a porcine blabber tissue and released about $14 \mathrm{ng}$ DTX in a $280 \mathrm{mT}$ magnetic field (two actuations) which is within the active range of DTX. However, depending on the application, the device design might require changes to suit the conditions.

To summarize, this implantable device offers controllable on demand drug administration and is capable of increasing the drug dosage by either more actuations or employing stronger magnetic field which both of them result in more released drug. The magnetic actuation eliminates the need of power and makes this method practical even with a permanent magnet. However, device might malfunction in an area with strong magnetic fields such as an MRI room.

\subsection{Future Work}

One perspective is to assess device performance inside a living biological environment, i.e.in vivo study. However, first of all, site of implantation and suitable device shape have to be determined. Other factors such as type and amount of medicine, prescribed release protocol, and duration of test are influential in device function. Depending on the implant duration, the 
biocompatibility of the device has to be inspected as well. Also, drug activity inside the reservoir in a long term test is to be studied.

This device could also be optimized by adjusting and enhancing the influential parameters such as type and concentration of the magnetic microparticles or the ratio of curing agent to base for PDMS. The latter element can alter the elasticity modulus of the PDMS, while, in this thesis, it was believed the least possible ratio corresponds to least stiffness [61]. However, the optimum ratio might be a different value in light of maximum overall contraction. Pores size and their effect on the sponge shrinkage may be studied as well. In this case, lower porosity increases the stiffness of sponge resulting in less deformation in a magnetic field, whereas, greater porosity implies less magnetic PDMS sections (more vacant space) which leads to less exerted force on the sponge in a magnetic field. Hence, the optimum case could be accomplished by considering all of these factors influence on the ultimate shrinkage of the sponge.

Employing a valve for the device aperture could prevent the background leakage, resulting in promoting the controllability of the device. However, this device has shown negligible and acceptable leakage rate due to its low amount. Although a one way valve can be a good candidate for this purpose, consequences of using a valve such as minimum required opening pressure, back pressure due to valve closure, and its durability can directly impinge on the release performance of the device. In order to improve the controllability of this device, a closed loop system can also be proposed by designating a biosensor for the device. 


\section{References}

[1] R. S. Langer, "New Methods of Drug Delivery,” Science (80-. )., vol. 249, no. 4976, pp. $1527-1533,1990$.

[2] V. Ranade, "Drug Delivery Oral Drug Systems Delivery," J Clin Phamacol, vol. 31, pp. 2-16, 1991.

[3] Y. H. Yun, B. K. Lee, and K. Park, "Controlled Drug Delivery: Historical perspective for the next generation," J. Control. Release, vol. 219, pp. 2-7, 2015.

[4] X. Pang, H. L. Du, H. Q. Zhang, Y. J. Zhai, and G. X. Zhai, "Polymer-drug conjugates: Present state of play and future perspectives," Drug Discov. Today, vol. 18, no. 23-24, pp. 1316-1322, 2013.

[5] J. M. Meerum Terwogt, W. W. ten Bokkel Huinink, J. H. Schellens, M. Schot, I. a Mandjes, M. G. Zurlo, M. Rocchetti, H. Rosing, F. J. Koopman, and J. H. Beijnen, "Phase I clinical and pharmacokinetic study of PNU166945, a novel water-soluble polymerconjugated prodrug of paclitaxel.," Anticancer. Drugs, vol. 12, no. 4, pp. 315-323, 2001.

[6] A. D. Conjugates, P. A. Vasey, S. B. Kaye, R. Morrison, C. Twelves, P. Wilson, R. Duncan, A. H. Thomson, L. S. Murray, T. E. Hilditch, T. Murray, S. Burtles, D. Fraier, and E. Frigerio, "Phase I Clinical and Pharmacokinetic Study of PK1 [ N - ( 2Hydroxypropyl ) methacrylamide Copolymer Doxorubicin ]: First Member of a New Class of Chemotherapeutic," vol. 5, no. January, pp. 83-94, 1999.

[7] H. Wu, L. Zhu, and V. P. Torchilin, "PH-sensitive poly(histidine)-PEG/DSPE-PEG copolymer micelles for cytosolic drug delivery," Biomaterials, vol. 34, no. 4, pp. 1213$1222,2013$. 
[8] R. Yang, A. V. Gorelov, F. Aldabbagh, W. M. Carroll, and Y. Rochev, “An implantable thermoresponsive drug delivery system based on Peltier device," Int. J. Pharm., vol. 447, no. 1-2, pp. 109-114, 2013.

[9] G. Vilar, J. Tulla-Puche, and F. Albericio, "Polymers for drug delivery systems," Curr. Drug Deliv., vol. 9, no. 4, pp. 367-94, 2012.

[10] R. Article, S. M. Yadav, A. K. Pareek, M. Kumar, and S. Gupta, "Osmotic drug delivery system: a new approach," vol. 2, no. 1, pp. 11-25, 2015.

[11] R. K. Verma, S. Garg, and F. Directions, "Current Status of Drug Delivery Technologies and Future Directions," Pharm. Technol., vol. 25, no. February, pp. 1-14, 2001.

[12] H. Rosen and T. Abribat, "The rise and rise of drug delivery.," Nat. Rev. Drug Discov., vol. 4, no. 5, pp. 381-385, 2005.

[13] J. C. Wright, S. Tao Leonard, C. L. Stevenson, J. C. Beck, G. Chen, R. M. Jao, P. A. Johnson, J. Leonard, and R. J. Skowronski, “An in vivo/in vitro comparison with a leuprolide osmotic implant for the treatment of prostate cancer," J. Control. Release, vol. 75, no. 1-2, pp. 1-10, 2001.

[14] W. Ryu, Z. Huang, F. B. Prinz, S. B. Goodman, and R. Fasching, "Biodegradable microosmotic pump for long-term and controlled release of basic fibroblast growth factor," $J$. Control. Release, vol. 124, no. 1-2, pp. 98-105, 2007.

[15] S. Herrlich, S. Spieth, S. Messner, and R. Zengerle, "Osmotic micropumps for drug delivery," Adv. Drug Deliv. Rev., vol. 64, no. 14, pp. 1617-1627, 2012.

[16] R. K. Verma and S. Garg, "Development and evaluation of osmotically controlled oral drug delivery system of glipizide," Eur. J. Pharm. Biopharm., vol. 57, no. 3, pp. 513-525, 2004. 
[17] G. Santus and R. W. Baker, "Osmotic drug delivery: a review of the patent literature," J. Control. Release, vol. 35, no. 1, pp. 1-21, 1995.

[18] J. T. Santini, M. J. Cima, and R. Langer, “A controlled-release microchip.," Nature, vol. 397, no. 6717, pp. 335-338, 1999.

[19] J. M. Maloney, S. A. Uhland, B. F. Polito, N. F. Sheppard, C. M. Pelta, and J. T. Santini, "Electrothermally activated microchips for implantable drug delivery and biosensing," $J$. Control. Release, vol. 109, no. 1-3, pp. 244-255, 2005.

[20] A. J. Chung, Y. S. Huh, and D. Erickson, “A robust, electrochemically driven microwell drug delivery system for controlled vasopressin release," Biomed. Microdevices, vol. 11, no. 4, pp. 861-867, 2009.

[21] S. Murdan, "Electro-responsive drug delivery from hydrogels," J. Control. Release, vol. 92, no. 1-2, pp. 1-17, 2003.

[22] Y. T. Yi, J. Y. Sun, Y. W. Lu, and Y. C. Liao, "Programmable and on-demand drug release using electrical stimulation,” Biomicrofluidics, vol. 9, no. 2, p. 022401, 2015.

[23] S. Ramanathan and L. H. Block, "The use of chitosan gels as matrices for electricallymodulated drug delivery," J. Control. Release, vol. 70, no. 1-2, pp. 109-123, 2001.

[24] O. Veiseh, J. W. Gunn, and M. Zhang, "Design and fabrication of magnetic nanoparticles for targeted drug delivery and imaging," Adv. Drug Deliv. Rev., vol. 62, no. 3, pp. 284$304,2010$.

[25] V. V. Mody, A. Cox, S. Shah, A. Singh, W. Bevins, and H. Parihar, "Magnetic nanoparticle drug delivery systems for targeting tumor," Appl. Nanosci., vol. 4, no. 4, pp. 385-392, 2013.

[26] K. Cai, Z. Luo, Y. Hu, X. Chen, Y. Liao, L. Yang, and L. Deng, "Magnetically triggered 
reversible controlled drug delivery from microfabricated polymeric multireservoir devices," Adv. Mater., vol. 21, no. 40, pp. 4045-4049, 2009.

[27] S. Rahimi, E. H. Sarraf, G. K. Wong, and K. Takahata, "Implantable drug delivery device using frequency-controlled wireless hydrogel microvalves," Biomed. Microdevices, vol. 13, no. 2, pp. 267-277, 2011.

[28] T. Y. Liu, S. H. Hu, K. H. Liu, D. M. Liu, and S. Y. Chen, "Study on controlled drug permeation of magnetic-sensitive ferrogels: Effect of Fe3O4 and PVA," J. Control. Release, vol. 126, no. 3, pp. 228-236, 2008.

[29] X. Zhao, J. Kim, C. a Cezar, N. Huebsch, K. Lee, K. Bouhadir, and D. J. Mooney, “Active scaffolds for on-demand drug and cell delivery.," Proc. Natl. Acad. Sci. U. S. A., vol. 108, no. 1, pp. 67-72, 2011.

[30] F. N. Pirmoradi, J. K. Jackson, H. M. Burt, and M. Chiao, "On-demand controlled release of docetaxel from a battery-less MEMS drug delivery device.," Lab Chip, vol. 11, no. 16, pp. 2744-2752, 2011.

[31] F. N. Pirmoradi, J. K. Jackson, H. M. Burt, and M. Chiao, "A magnetically controlled MEMS device for drug delivery: design, fabrication, and testing.," Lab Chip, vol. 11, no. 18, pp. 3072-3080, 2011.

[32] P. Zachkani, J. K. Jackson, F. N. Pirmoradi, and M. Chiao, “A cylindrical magneticallyactuated drug delivery device proposed for minimally invasive treatment of prostate cancer," RSC Adv., vol. 5, no. 119, pp. 98087-98096, 2015.

[33] M. Mustapić, M. S. Al Hossain, J. Horvat, P. Wagner, D. R. G. Mitchell, J. H. Kim, G. Alici, Y. Nakayama, and B. Martinac, "Controlled delivery of drugs adsorbed onto porous Fe3O4 structures by application of AC/DC magnetic fields," Microporous Mesoporous 
Mater., vol. 226, pp. 243-250, 2016.

[34] T. Hoare, J. Santamaria, G. F. Goya, S. Irusta, D. Lin, S. Lau, R. Padera, R. Langer, and D. S. Kohane, “A magnetically triggered composite membrane for on-demand drug delivery," Nano Lett., vol. 9, no. 10, pp. 3651-3657, 2009.

[35] R. Lo, P. Y. Li, S. Saati, R. N. Agrawal, M. S. Humayun, and E. Meng, "A passive MEMS drug delivery pump for treatment of ocular diseases," Biomed. Microdevices, vol. 11, no. 5, pp. 959-970, 2009.

[36] H. Gensler, R. Sheybani, P. Y. Li, R. Lo, S. Zhu, K. T. Yong, I. Roy, P. N. Prasad, R. Masood, U. K. Sinha, and E. Meng, "Implantable MEMS drug delivery device for cancer radiation reduction," Proc. IEEE Int. Conf. Micro Electro Mech. Syst., no. Di, pp. 23-26, 2010.

[37] C. Mousoulis, M. Ochoa, D. Papageorgiou, and B. Ziaie, "A skin-contact-actuated micropump for transdermal drug delivery," IEEE Trans. Biomed. Eng., vol. 58, no. 5, pp. 1492-1498, 2011.

[38] F. N. Pirmoradi, K. Ou, J. K. Jackson, K. Letchford, J. Cui, K. T. Wolf, F. Gräber, T. Zhao, J. A. Matsubara, H. Burt, M. Chiao, and L. Lin, "Controlled delivery of antiangiogenic drug to human eye tissue using a MEMS device," Proc. IEEE Int. Conf. Micro Electro Mech. Syst., pp. 1-4, 2013.

[39] B. P. Timko, M. Arruebo, S. A. Shankarappa, J. B. McAlvin, O. S. Okonkwo, B. Mizrahi, C. F. Stefanescu, L. Gomez, J. Zhu, A. Zhu, J. Santamaria, R. Langer, and D. S. Kohane, "Near-infrared-actuated devices for remotely controlled drug delivery.," Proc. Natl. Acad. Sci. U. S. A., vol. 111, no. 4, pp. 1349-54, 2014.

[40] S. R. Sirsi and M. A. Borden, "State-of-the-art materials for ultrasound-triggered drug 
delivery," Adv. Drug Deliv. Rev., vol. 72, pp. 3-14, 2014.

[41] K. J. Cha and D. S. Kim, “A portable pressure pump for microfluidic lab-on-a-chip systems using a porous polydimethylsiloxane (PDMS) sponge," Biomed. Microdevices, vol. 13, no. 5, pp. 877-883, 2011.

[42] M. G. King, A. J. Baragwanath, M. C. Rosamond, D. Wood, and A. J. Gallant, "Porous PDMS force sensitive resistors," Procedia Chem., vol. 1, no. 1, pp. 568-571, 2009.

[43] E. Pedraza, A. C. Brady, C. A. Fraker, and C. L. Stabler, "Synthesis of macroporous poly(dimethylsiloxane) scaffolds for tissue engineering applications," J. Biomater. Sci. Ed., vol. 24, no. 9, pp. 1041-1056, 2013.

[44] E. Pedraza, A. C. Brady, C. A. Fraker, R. D. Molano, S. Sukert, D. M. Berman, N. S. Kenyon, A. Pileggi, C. Ricordi, and C. L. Stabler, "Macroporous three-dimensional PDMS scaffolds for Extrahepatic Islet Transplantation," Cell Transplant., vol. 22, no. 7, pp. 1123-1125, 2013.

[45] J. Li, M. Zhang, L. Wang, W. Li, P. Sheng, and W. Wen, "Design and fabrication of microfluidic mixer from carbonyl iron-PDMS composite membrane," Microfluid. Nanofluidics, vol. 10, no. 4, pp.919-925, 2011.

[46] S. Bhattacharya, A. Datta, J. M. Berg, and S. Gangopadhyay, "Studies on surface wettability of poly(dimethyl) siloxane (PDMS) and glass under oxygen-plasma treatment and correlation with bond strength," J. Microelectromechanical Syst., vol. 14, no. 3, pp. 590-597, 2005.

[47] C. Ferlini, G. Scambia, M. Distefano, P. Filippini, G. Isola, a Riva, E. Bombardelli, a Fattorossi, P. Benedetti Panici, and S. Mancuso, "Synergistic antiproliferative activity of tamoxifen and docetaxel on three oestrogen receptor-negative cancer cell lines is mediated 
by the induction of apoptosis.," Br. J. Cancer, vol. 75, no. 6, pp. 884-891, 1997.

[48] T. M. Beer, W. C. P. B. A. Lowe, and W. D. Henner, "Original article Phase II study of weekly docetaxel in symptomatic androgen-independent prostate cancer," pp. 1273-1279, 2001.

[49] W. Schuette, S. Nagel, T. Blankenburg, C. Lautenschlaeger, K. Hans, E. W. Schmidt, I. Dittrich, H. Schweisfurth, L. F. Von Weikersthal, A. Raghavachar, A. Reißig, and M. Serke, "Phase III study of second-line chemotherapy for advanced non-small-cell lung cancer with weekly compared with 3-weekly docetaxel," J. Clin. Oncol., vol. 23, no. 33, pp. 8389-8395, 2005.

[50] V. J. Sinibaldi, M. A. Carducci, S. Moore-Cooper, M. Laufer, M. Zahurak, and M. A. Eisenberger, "Phase II evaluation of docetaxel plus one-day oral estramustine phosphate in the treatment of patients with androgen independent prostate carcinoma," Cancer, vol. 94, no. 5, pp. 1457-1465, 2002.

[51] J. Hong, S. H. Park, S. J. Choi, S. H. Lee, K. C. Lee, J. I. Lee, S. Y. Kyung, C. H. An, S. P. Lee, J. W. Park, S. H. Jeong, E. Nam, S. M. Bang, E. K. Cho, D. B. Shin, and J. H. Lee, "Nail toxicity after treatment with docetaxel: A prospective analysis in patients with advanced non-small cell lung cancer," Jpn. J. Clin. Oncol., vol. 37, no. 6, pp. 424-428, 2007.

[52] M. Shiota, A. Yokomizo, A. Takeuchi, K. Kiyoshima, J. Inokuchi, K. Tatsugami, and S. Naito, "Risk factors for febrile neutropenia in patients receiving docetaxel chemotherapy for castration-resistant prostate cancer," Support. Care Cancer, vol. 22, no. 12, pp. 32193226, 2014.

[53] D. Winther, D. M. Saunte, M. Knap, V. Haahr, and A. B. Jensen, "Nail changes due to 
docetaxel - A neglected side effect and nuisance for the patient," Support. Care Cancer, vol. 15, no. 10, pp. 1191-1197, 2007.

[54] Y. Yin, F. Cui, C. Mu, S. J. Chung, C. K. Shim, and D. D. Kim, "Improved solubility of docetaxel using a microemulsion delivery system: Formulation optimization and evaluation,” Asian J. Pharm. Sci., vol. 4, no. 6, pp. 331-339, 2009.

[55] Y. Po-Ying, "Mems-based anti-biofouling - mechanism, devices and application," University of British Columbia, 2009.

[56] D. P. Petrylak, C. M. Tangen, M. H. A. Hussain, P. N. Lara, J. A. Jones, M. E. Taplin, P. A. Burch, D. Berry, C. Moinpour, M. Kohli, M. C. Benson, E. J. Small, D. Raghavan, and E. D. Crawford, "Docetaxel and estramustine compared with mitoxantrone and prednisone for advanced refractory prostate cancer.," N. Engl. J. Med., vol. 351, no. 15, pp. 1513-20, 2004.

[57] J. Horti, A. Pluzanska, K. N. Chi, S. Oudard, C. Théodore, M. A. Rosenthal, D. Ph, and M. A. Eisenberger, "Docetaxel plus Prednisone or Mitoxantrone plus Prednisone for Advanced Prostate Cancer," pp. 1502-1512, 2004.

[58] J. Verbraecken, P. Van De Heyning, W. De Backer, and L. Van Gaal, "Body surface area in normal-weight, overweight, and obese adults. A comparison study," Metabolism., vol. 55, no. 4, pp. 515-524, 2006.

[59] P. Zahedi, R. De Souza, M. Piquette-Miller, and C. Allen, "Chitosan-phospholipid blend for sustained and localized delivery of docetaxel to the peritoneal cavity," Int. J. Pharm., vol. 377, no. 1-2, pp. 76-84, 2009.

[60] P. Zachkani, "A minimally-invasive mems drug delivery device for the treatment of prostate cancer," University of British Columbia, 2014. 
[61] T. K. Kim, J. K. Kim, and O. C. Jeong, "Measurement of nonlinear mechanical properties of PDMS elastomer," Microelectron. Eng., vol. 88, no. 8, pp. 1982-1985, 2011. 


\section{Appendices}

\section{Appendix A : Drug Release Actuation Setup}

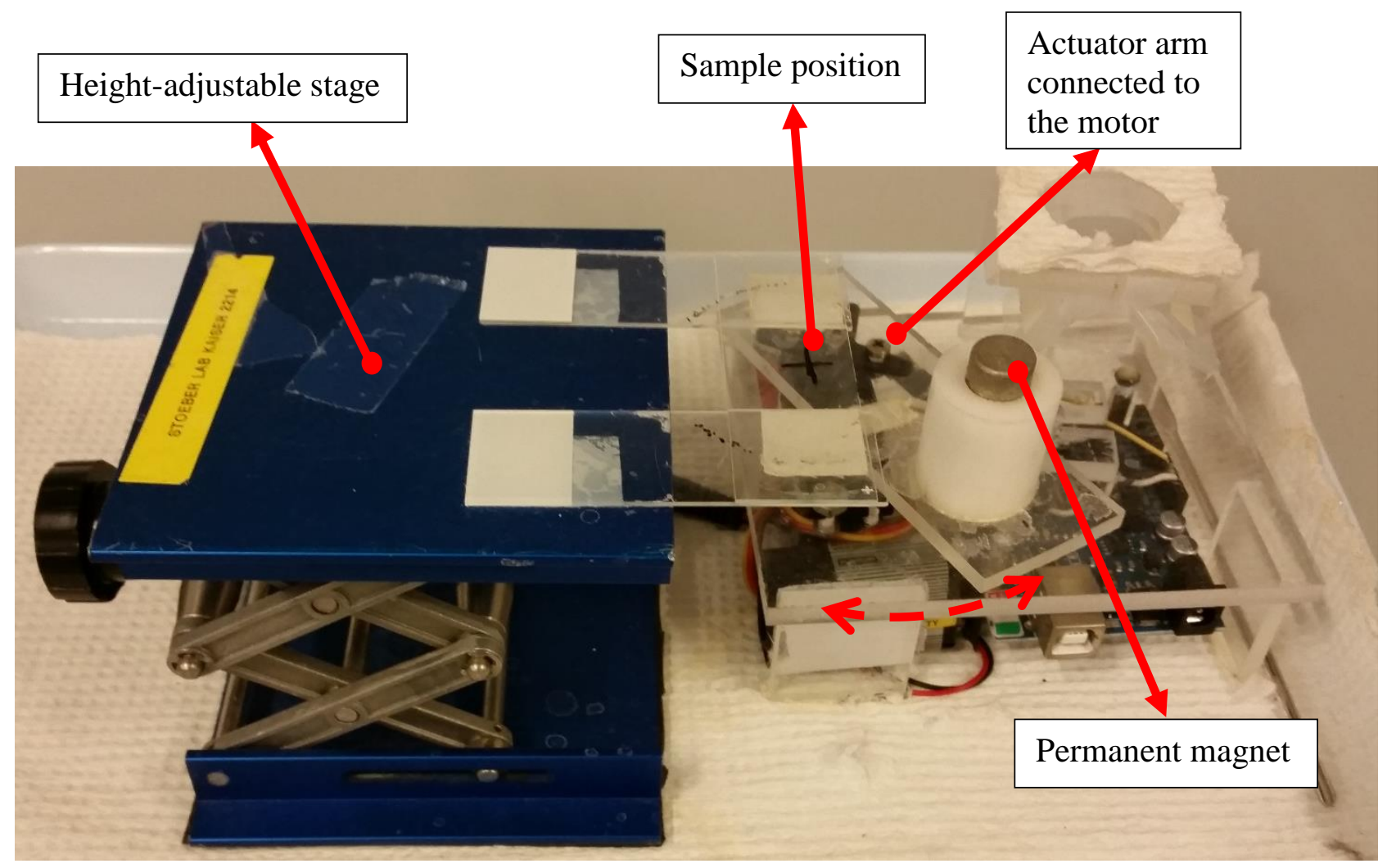

Figure 20-Actuation setup for drug release test 


\section{Appendix B : Spectrophotometer}

5 samples containing different known methylene blue concentration were prepared and their absorbance were measured at a wavelength of $662 \mathrm{~nm}$, using the UV-Vis spectrophotometer (50 BIO, Varian Medical Systems Inc., Palo Alto, CA, USA). Later a curve was fitted to the obtained data and its equation was used to convert the measured absorbance of subsequent samples to the concentration. Since the volume of the medium was a determined value $(4 \mathrm{ml})$, the amount of released methylene blue was achieved once the concentration was computed.

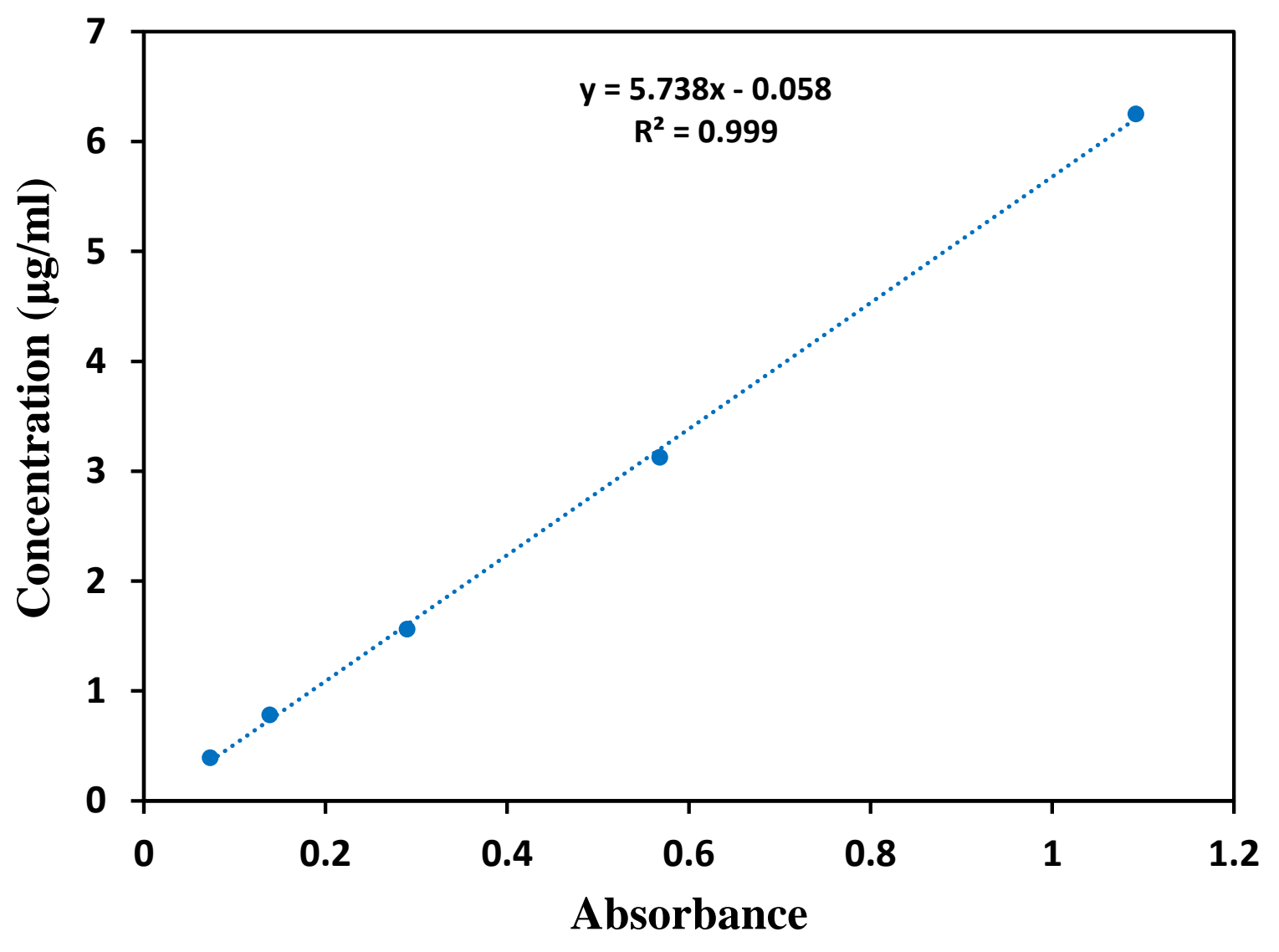

Figure 21- Standard curve obtained for the MB test 


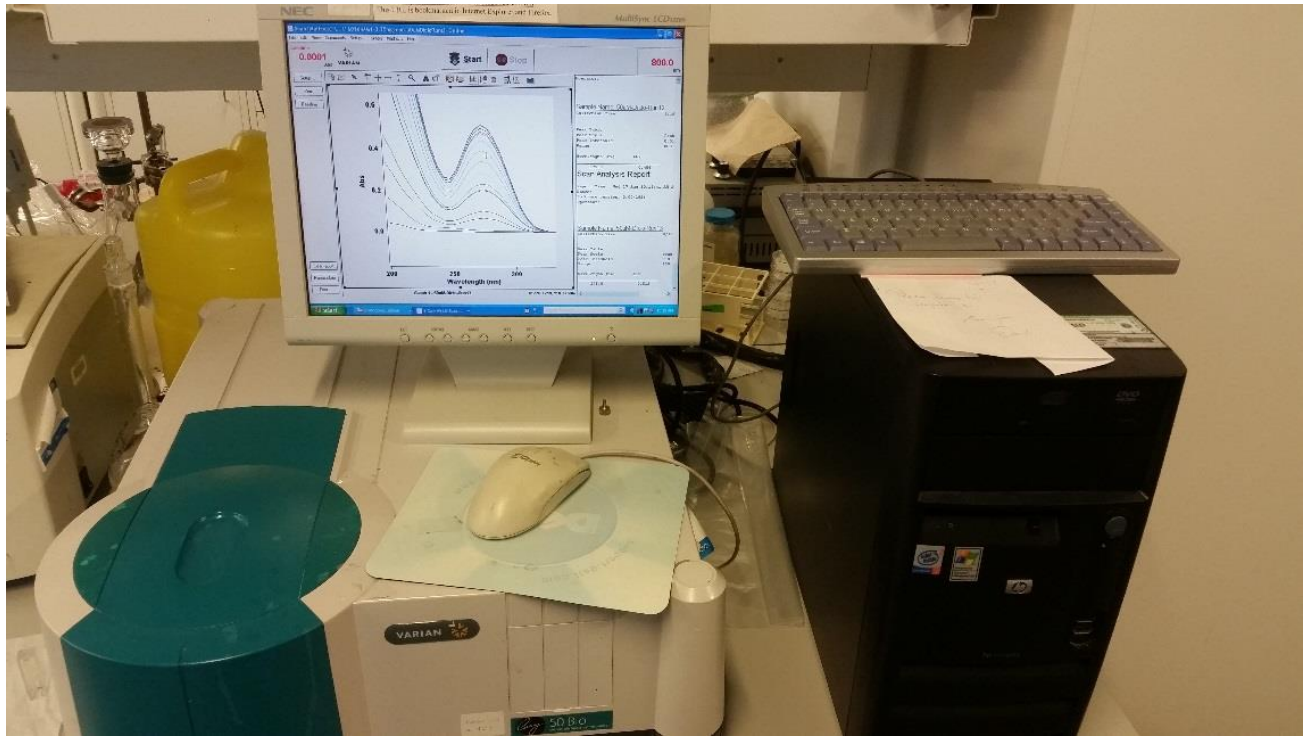

Figure 22- UV-Vis spectrophotometer (50 BIO, Varian Medical Systems Inc., Palo Alto, CA, USA) 